\title{
Modeling Spontaneous Activity in the Developing Spinal Cord Using Activity-Dependent Variations of Intracellular Chloride
}

\author{
Cristina Marchetti, ${ }^{1,2}$ Joel Tabak, ${ }^{1}$ Nikolai Chub, ${ }^{1}$ Michael J. O'Donovan, ${ }^{1}$ and John Rinzel ${ }^{2,3}$ \\ ${ }^{1}$ Laboratory of Neural Control, National Institute of Neurological Disorders and Stroke, National Institutes of Health, Bethesda, Maryland 20892, and \\ ${ }^{2}$ Center for Neural Science and ${ }^{3}$ Courant Institute of Mathematical Sciences, New York University, New York, New York 10003
}

We investigated how spontaneous activity is generated in developing, hyperexcitable networks. We focused our study on the embryonic chick spinal cord, a preparation that exhibits rhythmic discharge on multiple timescales: slow episodes (lasting minutes) and faster intraepisode cycling $(\sim 1 \mathrm{~Hz}$ frequency). For this purpose, we developed a mean field model of a recurrent network with slow chloride dynamics and a fast depression variable. We showed that the model, in addition to providing a biophysical mechanism for the slow dynamics, was able to account for the experimentally observed activity. The model made predictions on how interval and duration of episodes are affected when changing chloride-mediated synaptic transmission or chloride flux across cell membrane. These predictions guided experiments, and the model results were compared with experimental data obtained with electrophysiological recordings. We found agreement when transmission was affected through changes in synaptic conductance and good qualitative agreement when chloride flux was varied through changes in external chloride concentration or in the rate of the $\mathrm{Na}^{+}-\mathrm{K}^{+}-2 \mathrm{Cl}^{-}$cotransporter. Furthermore, the model made predictions about the time course of intracellular chloride concentration and chloride reversal potential and how these are affected by changes in synaptic conductance. Based on the comparison between modeling and experimental results, we propose that chloride dynamics could be an important mechanism in rhythm generation in the developing chick spinal cord.

Key words: spontaneous activity; network; development; chloride; spinal cord; modeling

\section{Introduction}

Spontaneous activity is an important property of the developing nervous system (Katz and Shatz, 1996; O'Donovan, 1999). It usually involves episodes of rhythmic discharge, during which many neurons are activated synchronously, separated by longer quiescent periods. Although the phenomenon is not fully understood, it may influence several aspects of neuronal or synaptic development (Gu and Spitzer, 1995; Katz and Shatz, 1996; Li et al., 1996; Zhang and Poo, 2001; Borodinsky et al., 2004).

It has been proposed that spontaneous activity is generated in hyperexcitable networks that are subject to some form of activitydependent depression (Feller et al., 1999; O’Donovan, 1999; Opitz et al., 2002). During development, the classical inhibitory neurotransmitters GABA and glycine can be functionally excitatory because the chloride equilibrium potential is above rest as a result of an elevated intracellular chloride concentration (Chub and O'Donovan, 2001) (for review, see Ben Ari, 2002). This leads

Received 0ct. 14, 2004; revised Feb. 4, 2005; accepted Feb. 19, 2005.

C.M. was supported as a Fogarty Fellow by National Institute of Neurological Disorders and Stroke. C.M. and J.R. express appreciation for the hospitality of the Center for Neural Science, New York University and the Laboratory of Biological Modeling, National Institute of Diabetes and Digestive and Kidney Diseases, and National Institutes of Health for hosting them as part-time guest researchers.

Correspondence should be addressed to John Rinzel, Center for Neural Science, New York University, 4 Washington Place, Room 809, New York, NY 10003. E-mail: rinzel@cns.nyu.edu.

DOI:10.1523/JNEUROSCI.4290-04.2005

Copyright $\odot 2005$ Society for Neuroscience $\quad$ 0270-6474/05/253601-12\$15.00/0 to a predominance of excitatory synapses that renders developing networks hyperexcitable. We proposed that, in the chick embryo spinal cord, a fast form of depression, operating on a timescale $<1 \mathrm{~s}$, regulates cycle generation within the episodes, whereas a much slower form of depression (timescale of minutes) regulates the expression of episodes (Fedirchuk et al., 1999; Tabak et al., 2000). This prolonged time course of the slow depression (from a few minutes to more than $30 \mathrm{~min}$ ) is highly unusual and cannot be explained by traditional forms of synaptic depression (e.g., transmitter depletion) or channel inactivation, which are much faster (Varela et al., 1997).

Recently, it was found that intracellular chloride concentration undergoes significant changes during spontaneous activity in the chick cord (Chub and O'Donovan, 2001). After an episode, the chloride equilibrium potential falls by $\sim 10 \mathrm{mV}$, corresponding to a decline of intracellular chloride of $\sim 15 \mathrm{~mm}$. The intracellular chloride concentration is restored during the interepisode interval, presumably attributable to inward chloride pumping via the developmentally regulated cationic-chloride cotransporter NKCC1 (for review, see Delpire, 2000). The effect of chloride extrusion during an episode is to reduce the driving force for chloride-mediated synaptic potentials and hence network excitability. During the subsequent interepisode interval, intracellular chloride recovers, leading to a progressive increase in network excitability. Moreover, the observation that network activity is essentially unchanged in the presence of glutamatergic 
and cholinergic transmitter antagonists suggests the importance of chloride-mediated transmission (Chub and O'Donovan, 1998).

Collectively, these observations have led to the proposal that the slow depression controlling the interepisode interval might be a manifestation of the reduction of intracellular chloride that accompanies the episode (Chub and O'Donovan, 2001). In this paper, we investigated whether activity-dependent changes of intracellular chloride can indeed result in the expression of spontaneous episodes. We developed a model network with recurrent excitatory connections that was subject to activity-dependent changes of intracellular $\mathrm{Cl}^{-}$concentration. We show that the behavior of the model reproduces the observed pattern of episodic activity, and we make several predictions, which we test experimentally.

Parts of this work have been published previously in abstract form (Marchetti et al., 2003).

\section{Materials and Methods}

Theoretical. We develop and use a mean field three-variable model for a population of "functionally excitatory" cells. The primary activity variable is mean membrane potential, $V(t)$, considered to be temporally averaged over the timescale of a few spikes (Pinto et al., 1996). This $V$ determines the instantaneous firing rate $f(V)$, represented by the sigmoid function and scaled by the maximal firing rate as follows:

$$
f(V)=1 /\left[1+\exp \left(\left(\theta_{f}-V\right) / k_{f}\right)\right],
$$

where $k_{f}$ defines the steepness of the sigmoid, and $\theta_{f}$ corresponds to $V$ at which half-maximal firing occurs. This threshold is an approximate measure of firing threshold (chosen to be $-43 \mathrm{mV}$ from Chub and O'Donovan, 2001). The other two dynamic variables are as follows: $d$, synaptic depression (the fraction of synapses not affected by fast synaptic depression), and $\mathrm{Cl}_{i}$, the intracellular $\mathrm{Cl}^{-}$concentration that affects firing and coupling through its influence on the GABAergic synaptic reversal potential $E_{\mathrm{Cl}}$. The following equations describe the network dynamics; the variables represent averages over the population and describe the state of the "typical cell":

$$
\begin{gathered}
\tau_{V} \dot{V}=-\left[\left(V-V_{\text {rest }}\right)+I_{\text {syn }} / g_{\text {leak }}\right], \\
\tau_{d} \dot{d}=d_{\infty}(V)-d \\
\dot{C} l_{i}=\left[I_{\text {syn }} / F+R_{\text {co-trans }}\right] / \mathrm{Vol}_{\mathrm{Cl}},
\end{gathered}
$$

where $\dot{X}$ denotes time derivative $d X / d t$.

The first equation corresponds to the current-balance condition in a cell. In this idealization, spikes have been averaged out because, most of the time, a cell is sitting near rest or driven by strong synaptic inputs to depolarized levels. Therefore, only an effective leakage term remains from the intrinsic spike-generating mechanisms. The time constant $\tau_{V}$ is not merely the membrane time constant. It incorporates the average effect of recruitment in the network (see a few paragraphs below), the membrane time constant, axonal conduction velocity, and synaptic/dendritic delays.

The synaptic current is defined by:

$$
I_{\text {syn }}=g_{\text {syn }} \times d \times f(V) \times\left(V-E_{\mathrm{Cl}}\right),
$$

where the effective synaptic conductance is proportional to the maximal level, $g_{\text {syn }}$ (if no synapses are depressed and all cells are firing at the maximal rate), times the fraction of nondepressed synapses $d$ and the firing rate $f(V)$. The synaptic reversal potential $E_{\mathrm{Cl}}$ satisfies the Nernst equation:

$$
E_{\mathrm{Cl}}=(R T / F) \ln \left(\mathrm{Cl}_{i} /\left[\mathrm{Cl}^{-}\right]_{\mathrm{ext}}\right),
$$

where $R T / F$ is a constant $(R T / F \simeq 25$ at room temperature). In control conditions, external $\mathrm{Cl}^{-}$concentration $\left(\left[\mathrm{Cl}^{-}\right]_{\text {ext }}\right)$ was set to $150 \mathrm{~mm}$.

Equations 2 and 3 describe the dynamics of the variables that reduce synaptic throughput within the network on two different timescales. Synaptic depression on a fast timescale is represented by $d$; its interaction with $V$ gives rise to intraepisode cycling (Tabak et al., 2000), with cycle periods of hundreds of milliseconds. Because $d$ decreases with activity, $d_{\infty}(V)$ is a decreasing function, which we take to be sigmoidal:

$$
d_{\infty}(V)=1 /\left[1+\exp \left(\left(\theta_{\mathrm{d}}-V\right) / k_{d}\right)\right], \quad k_{d}<0 .
$$

Because we averaged over spikes, $d_{\infty}(V)$ incorporates the dependence of the firing rate on $V$.

On the slower timescale, the chloride dynamics are responsible for episode generation that occurs with intervals of minutes. The slowly changing intracellular chloride concentration, $\mathrm{Cl}_{i}$, underlies slow depression through its effect on the synaptic driving potential, without a direct effect on synaptic conductance. In accordance with previous experimental data (Chub and O'Donovan, 2001), intracellular chloride is on average extruded during an episode (through chloride-selective ligand-gated synaptic channels) and recovers to pre-episode levels during the interepisode interval, through an active mechanism that transports chloride inward, i.e., the NKCC cotransporter. Equation 3, the $\mathrm{Cl}^{-}$-balance equation, embodies these two mechanisms. It states that the rate at which $\mathrm{Cl}_{i}$ changes depends on two flux terms. The electrical current $I_{\text {syn }}$ when divided by Faraday's constant $\left(F=9.6485 \times 10^{4} \mathrm{C} / \mathrm{mol}\right)$ is converted to molecular flux (moles per second). Because $V<E_{\mathrm{Cl}}$, this term is negative, corresponding to efflux. The NKCC cotransporter pumps $\mathrm{Cl}^{-}$inward with the rate $R_{\text {co-trans }}$ (moles per second). These fluxes, when divided by the effective intracellular volume for $\mathrm{Cl}^{-}$, equal their contributions to the change (per second) in chloride concentration (molar units). We note that, because this cotransporter is non-electrogenic, it produces no net electrical current, and, therefore, the current-balance Equation 1 has no term for it.

In our model, $g_{\text {leak }}$ represents the mean resting conductance for ions other than $\mathrm{Cl}^{-}$. This is why there is no explicit term for $\mathrm{Cl}^{-}$leakage current in Equation 3. However, because $f\left(V_{\text {rest }}\right)$ is non-zero, there is some $\mathrm{Cl}^{-}$current at rest. This could be thought to include a leakage component as well as spontaneous GABA-mediated current and spontaneously firing cells (Chub and O'Donovan, 2001); our idealized model does not distinguish these factors.

Currently, we have estimates for only a few cellular parameter values. The resting potential $\left(V_{\text {rest }}=-60 \mathrm{mV}\right)$ and the leak conductance ( $g_{\text {leak }}=3 \mathrm{nS}$ ) were estimated from perforated-patch recordings (Chub and O'Donovan, 2001). Effective $\mathrm{Cl}^{-}$-handling volume $\left(\mathrm{Vol}_{\mathrm{Cl}}=0.6 \times\right.$ $10^{-9} \mathrm{~cm}^{3}$ ) was chosen with typical anatomical length scales in mind; in the case of a sphere, it corresponds to a diameter of $\sim 10 \mu \mathrm{m}$. We first estimated $R_{\text {co-trans }}$ by noting that a 10 min interepisode interval increases intracellular chloride by $15 \mathrm{~mm}$ (Chub and O'Donovan, 2001). This gave us a value of $15 \times 10^{-6} \mathrm{pmol} / \mathrm{s}$. This value was increased because $I_{\text {syn }}$ is small but non-zero during the interepisode interval (Chub and O'Donovan, 2001), thereby creating an outward flux of $\mathrm{Cl}^{-}$. Because we used younger embryos than Chub and O'Donovan (2001), they exhibited shorter interepisodes intervals ( $\sim 5 \mathrm{~min})$. For these reasons, we chose $R_{\text {co-trans }}=12 \times 10^{-5} \mathrm{pmol} / \mathrm{s}$, which resulted in interepisode intervals of $\sim 3$ min (for $g_{\text {syn }}=30 \mathrm{pS}$ ) in the model.

We use absolute values for quantities such as $g_{\text {syn }}$ and $R_{\text {co-trans. }}$. Had we used per unit area densities instead, the associated flux terms might have different functional areas. Our model does not distinguish these possibilities.

In Equation $4, g_{\text {syn }}$, the maximal total synaptic conductance of a cell, was chosen to be in the range of $18-36 \mathrm{nS}$ and was varied in some figures. This range is consistent with estimates of GABAergic conductance of $\sim 15 \mathrm{nS}$ (Chub and O'Donovan, 2001); our range for the model is higher to account for the glycinergic component of the $\mathrm{Cl}^{-}$-based synaptic current. The values of $\tau, \theta$, and $k$ that we used in the simulations are given in Table 1. For finite values of $V, f(V)$ is never zero, i.e., there is always a minimal level of background activity or fraction of spontaneously active neurons in the network.

How do we choose values for the parameters $\tau_{V}$ and $\tau_{d}$ ? Because we do not have a mathematical derivation of our $V$ formulation of the mean field model, we rely on a rough estimate for what we think is the major 


\section{Table 1. Parameter values used in simulations}

\begin{tabular}{llll}
\hline & $\tau(\mathrm{s})$ & $k(\mathrm{mV})$ & $\theta(\mathrm{mV})$ \\
\hline$V$ & $\tau_{V}=0.1$ to 0.2 & & \\
$d_{\infty}(V)$ & $\tau_{d}=0.6$ & $k_{d}=2$ & $\theta_{d}=-45$ \\
$f(V)$ & & $k_{f}=-3$ & $\theta_{f}=-43$ \\
\hline
\end{tabular}
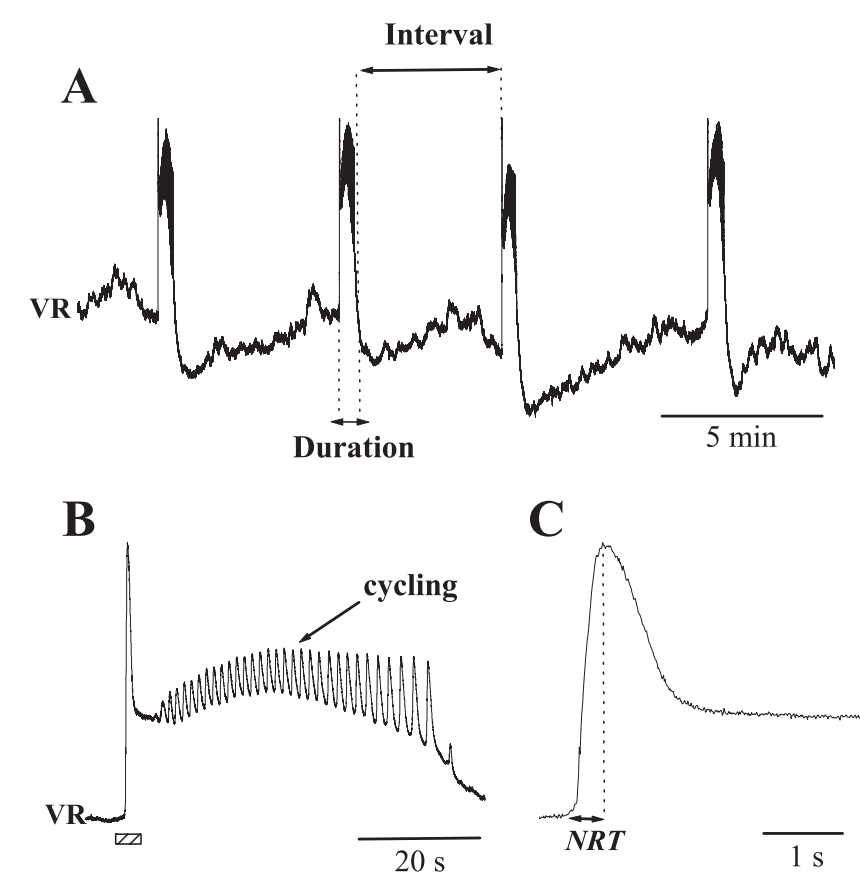

Figure 1. A, Example of spontaneous rhythmic activity in an E10 chick embryo spinal cord, recorded from a VR. $\boldsymbol{B}$, Expansion of a single episode (third one from the trace in $\boldsymbol{A}$ ), showing the presence of intraepisode cycling. $\boldsymbol{C}$, Expansion of the first peak (from $\boldsymbol{B}$, indicated by the bottom bar). Network recruitment time was measured as the time between the onset and the peak of ventral root activity.

contributor to the effective network time constant $\tau_{V}$. It sets the timescale for recruitment of cells throughout the network, i.e., the time it takes the cells in the distributed network to respond to changes of an exogenous or endogenous input. We estimated the recruitment time empirically as the time it takes for the ventral root (VR) slow potential to rise from baseline to the first peak of an episode (Fig. 1C, NRT). In our experimental conditions (i.e., in the presence of glutamatergic and cholinergic blockers), the average "network recruitment time" NRT was $570 \pm 80 \mathrm{~ms}(n=11)$. Therefore, because this value is a few multiples of $\tau_{V}$, we chose a value for $\tau_{V}$ on the order of 100-200 ms. In some other neural systems, the timescale of synaptic depression $\tau_{d}$ has been estimated to be on the scale of hundreds of milliseconds (Chance et al., 1998). These estimates provide the opportunity for cycle periods in the desired range. In our model, the values for $\tau_{d}$ and $\tau_{V}$ are further constrained by the fact that the output must show fast cycling at a frequency comparable with observed values. In Results, we will see that the behavior of the model in this regard is quite sensitive to the ratio $\tau_{d} / \tau_{v}$.

The model equations were implemented within XPPAUT (freely available software by G. B. Ermentrout, http://www.pitt.edu/ phase/), an interactive package for numerically solving and analyzing differential equations, which also includes a tool (AUTO) for computing bifurcation diagrams. Simulations were performed using the Gear or the RungeKutta method, with a time step of $0.001 \mathrm{~s}$ : we confirmed that this gave good accuracy by verifying that results were insensitive to an additional decrease in the time step.

Experimental. Experiments were performed on the isolated spinal cord of embryonic day 9 (E9) to E11 chick embryos. The spinal cord was dissected out in cold $\left(15^{\circ} \mathrm{C}\right)$, oxygenated $\left(95 \% \mathrm{O}_{2}, 5 \% \mathrm{CO}_{2}\right)$ Tyrode's solution containing the following (in $\mathrm{mm}$ ): $139 \mathrm{NaCl}, 3 \mathrm{KCl}, 17 \mathrm{NaHCO}_{3}$,
12 glucose, $3 \mathrm{CaCl}_{2}$, and $1 \mathrm{MgCl}_{2}$. The solution was then allowed to reach room temperature, and, at that point, the preparation was transferred to a recording chamber. Recordings were started at least $2 \mathrm{~h}$ after heating the bath solution at $28^{\circ} \mathrm{C}$. The superfusing Tyrode's solution was then kept at $28^{\circ} \mathrm{C}$ for the duration of experiments. The majority of experiments were performed in the presence of glutamatergic and cholinergic blockers (Chub and O'Donovan, 1998) because we were focusing on the $\mathrm{Cl}^{-}\left(E_{\mathrm{Cl}}\right)$ mechanism for episode termination and recovery. For this reason, $\left[\mathrm{K}^{+}\right]_{\mathrm{o}}$ was raised to $5 \mathrm{~mm}$, minimizing the slowing of spontaneous episodes that occurs when these blockers are used, without altering the characteristics of the activity (Chub and O'Donovan, 1998). Glutamatergic and cholinergic blockers were used at the following concentrations: $50 \mu \mathrm{M} \mathrm{AP}-5$, 5-20 $\mu \mathrm{M}$ 6-cyano-7-nitroquinoxaline-2,3-dione (CNQX), $5 \mu \mathrm{M}$ atropine, $50 \mu \mathrm{M}$ mecamylamine, and $50 \mu \mathrm{M}$ dihydro- $\beta$-erythroidine $(\mathrm{DH} \beta \mathrm{E})$.

Neuronal activity was recorded with tight-fitting suction electrodes from VRs or muscle nerves. Signals were amplified with high-gain direct current amplifiers [model 3000 (A-M Systems, Carlsborg, WA) or DAM 50 (World Precision Instruments, Sarasota, FL)]. A typical extracellular recording is shown in Figure 1A: the voltage recorded from one VR sharply rises when an episode begins and decays on a slower timescale. Intraepisode cycling is visible in Figure $1 B$, in which one episode is shown at an expanded time base. We measured episode duration and the interepisode interval that is defined as the time between the end of an episode and the beginning of the following one (Fig. $1 \mathrm{~A}$ ).

Electrical stimulation $(0.5 \mathrm{~ms}$ pulses at $5-10 \mu \mathrm{A})$ was delivered to the cord through the ventrolateral funiculus (VLF) and was used to monitor the effect of drugs that decrease GABAergic activity. Because these experiments were performed in the presence of glutamatergic and cholinergic blockers, we assumed that the responses we were recording were predominantly mediated by GABA and glycine (Chub and O'Donovan, 1998). We measured the early part of the evoked potential, i.e., within 5 $\mathrm{ms}$ of the onset, to minimize contributions from polysynaptic pathways. We compared the averages from at least 10 responses obtained under control conditions and $10 \mathrm{~min}$ after bicuculline application.

Whole-cell voltage-clamp recordings ("blind patch") were made with an Axopatch 200B amplifier (Axon Instruments, Union City, CA) from ventral horn neurons located in spinal segments LS1-LS3. Micropipettes were pulled from thin-walled glass capillaries (TW 100-3; World Precision Instruments) with a Flaming/Brown puller (P-80/PC; Sutter Instruments, Novato, CA). Tip resistances in the extracellular solution were 4-6 M $\Omega$. GABAergic currents were recorded in modified Tyrode's solution containing the following (in $\mathrm{mm}$ ): $129 \mathrm{NaCl}, 5 \mathrm{KCl}, 17 \mathrm{NaHCO}_{3}, 1$ $\mathrm{MgCl}_{2}, 0.5 \mathrm{MnCl}_{2}, 12$ glucose, 10 tetraethylammonium (TEA)-acetate, 5 $\mathrm{CsCl}$, and 0.001 TTX. To separate indirect effects of the bumetanide on GABAergic conductance, which arise from changing the intracellular $\mathrm{Cl}^{-}$concentration after blockade of the $\mathrm{Na}^{+}-\mathrm{K}^{+}-2 \mathrm{Cl}^{-}$cotransport (NKCC1), we used intracellular solution with high $\mathrm{Cl}^{-}$concentration containing the following (in mM): $140 \mathrm{CsCl}, 10 \mathrm{HEPES}, 1.1$ EGTA, 1 $\mathrm{MgCl}_{2}, 0.1 \mathrm{CaCl}_{2}, 4 \mathrm{Na}_{2} \mathrm{ATP}, 0.5 \mathrm{Na}-\mathrm{GTP}, 1$ phosphocreatine, and 10 TEA-acetate. This solution, in contrast to recent perforated-patch recordings (Sung et al., 2000), clamps the intracellular $\mathrm{Cl}^{-}$concentration and therefore allows measurement of the effects of the NKCC blocker bumetanide on GABA currents, unaccompanied by changes in the intracellular $\mathrm{Cl}^{-}$concentration.

All signals were digitized through a Digidata 1322A, stored, and analyzed with pClamp 8 software (Axon Instruments). Voltage-ramp protocols, for measurements and analysis of GABA currents, were similar to that published recently (Chub and O'Donovan, 2001).

To maintain constant osmolarity in solutions in which the $\left[\mathrm{Cl}^{-}\right]_{\mathrm{ext}}$ was changed ( +15 or $-15 \mathrm{~mm}$ ), we raised osmolarity by $30 \mathrm{mOsm}$ in all experiments of this type. In control Tyrode's solution $\left(\left[\mathrm{Cl}^{-}\right]_{\mathrm{ext}}=152\right.$ $\mathrm{mM}), 30 \mathrm{~mm}$ sucrose was added. In the case of $\left[\mathrm{Cl}^{-}\right]_{\mathrm{ext}}=137 \mathrm{mM}, 15 \mathrm{~mm}$ $\mathrm{NaCl}$ in the Tyrode's solution was replaced with an equal amount of Na-propionate, and $30 \mathrm{~mm}$ sucrose was also added. Finally, for $\left[\mathrm{Cl}^{-}\right]_{\mathrm{ext}}=167 \mathrm{~mm}$, only $15 \mathrm{~mm}$ cholene- $\mathrm{Cl}$ was added. For these experiments, glutamatergic blockers ( $50 \mu \mathrm{M}$ AP-5 and $20 \mu \mathrm{M}$ CNQX) were also added to all bath solutions. Receptor antagonists and bumetanide were bath applied. In whole-cell experiments, GABA was locally applied from 
micropipettes as described previously (Chub and O'Donovan, 2001) by using custom made picospritzer. CNQX, D-amino-phosphonovalerate (AP-5), DH $\beta \mathrm{E}$, mecamylamine, atropine, bicuculline, dehydroepiandrosterone sulfate (DHEAS), bumetanide, and other chemicals were purchased from Sigma (St. Louis, MO). Significance of data were tested with ANOVA or $t$ tests with an accepted level of significance of 0.05 .

\section{Results}

In this study, we developed an idealized network model with recurrent functionally excitatory, synaptic connections and used it to show that slow, activity-dependent variations in the chloride reversal potential, $E_{\mathrm{Cl}}$, produce episodes of activity. The firing rate-like framework of our model provides a plausible approximation to the actual network behavior because spontaneous activity generated by the spinal cord does not depend on the details of network architecture (Ho and O'Donovan, 1993; Chub and O'Donovan, 1998). Furthermore, the model does not require details of the connectivity or biophysical properties of individual spinal neurons (Tabak et al., 2000, 2001).

Our previous ad hoc model exhibited spontaneous switching between two steady states (a low-activity stable one and a highactivity oscillatory one), qualitatively reproducing the experimentally observed rhythmicity, through the interaction of three dynamic variables: an activity variable $(a)$, which represented the average firing rate $[f(V)$ in the present model]; a fast depression variable $(d)$ that acted to limit activity within an episode; and a slow depression variable $(s)$ responsible for terminating an episode and recovering on the timescale of the interepisode interval (Tabak et al., 2000, 2001). Depression variables appeared in the model as activity-dependent multiplicative factors that modulated synaptic input to a cell but were not related to biophysical observable quantities.

In the present work, we propose that intracellular chloride concentration, $\mathrm{Cl}_{i}$, is the physiological realization of the slow depression variable and that $\mathrm{Cl}^{-}$(hence, $E_{\mathrm{Cl}}$ ) dynamics are, at least in part, the biophysical mechanism that accounts for synaptic depression in the cord. This formulation is less ad hoc, incorporating semiquantitative estimates for some biophysical parameters. The activity-like variable is $V$, mean membrane potential, temporally averaged over the timescale of a few spikes; synaptic depression, $d$, on the fast timescale of cycling is like that in our previous model (Tabak et al., 2000, 2001). The mathematical formulation for this three-variable model is given in Materials and Methods (Eqs. 1-5). We show that our model is able to account for the generation of spontaneous episodic activity with intraepisode cycling, as observed in the chick spinal cord. In addition, we used the model to make several predictions and test them by manipulating experimentally accessible parameters, such as GABAergic conductances and $\left[\mathrm{Cl}^{-}\right]_{\text {ext }}$. By comparing model predictions and experimental results, we conclude that $\mathrm{Cl}^{-}$dynamics is a plausible mechanism contributing to episode generation in the developing spinal cord.

\section{Episodic behavior in the model}

Figure 2 demonstrates the behavior of our model and its ability to generate multicycle episodes, in a manner similar to our previous model. Between episodes, most cells are silent and most synapses are inactive, and there is a slow increase in mean membrane potential $V$ (Fig. $2 A$ ) as $E_{\mathrm{Cl}}$ is being restored by $\mathrm{Cl}^{-}$influx (transported by NKCC1) (Fig. $2 B$ ). The transport rate $R_{\text {co-trans }}$ determines the timescale of the interepisode interval. When a certain threshold for $\mathrm{Cl}_{i}$ is reached, an episode is initiated and $\mathrm{Cl}^{-}$flux reverses (Fig. $2 \mathrm{~B}$ ). During an episode, membrane potential is

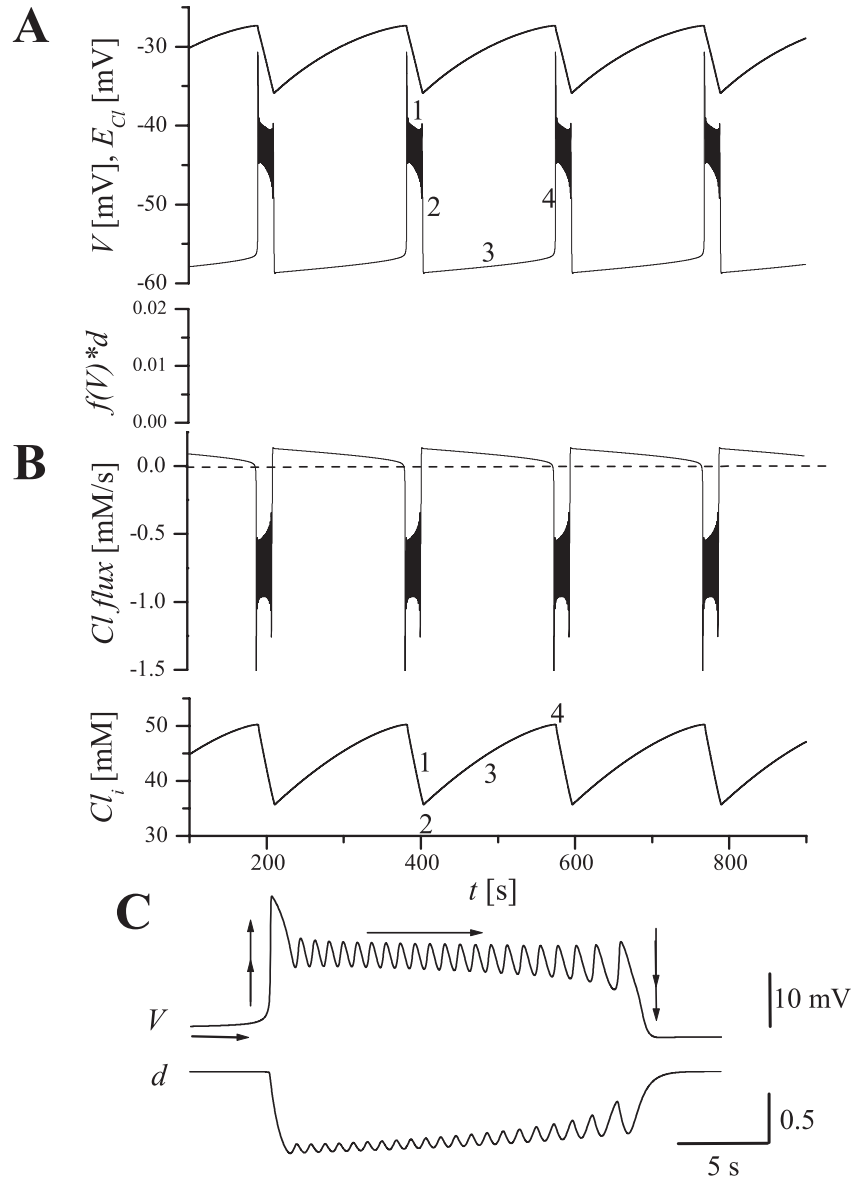

Figure 2. Episodic activity generated by the model. $A$, Time courses of $V$ and $E_{\mathrm{Cl}}$ (top traces). $E_{C I}$ decreases during an episode and recovers during the interepisode interval. Note that $V$ is always lower than $E_{\mathrm{Cl}}$. Bottom trace is time course of fraction of $\mathrm{Cl}^{-}$conductance, showing slow rise during interepisode phase (clipped during episode for better visualization). $\boldsymbol{B}$, Time course of $\mathrm{Cl}^{-}$flux and $\mathrm{Cl}_{i} \mathrm{Cl}^{-}$flux is positive during silent phases (influx attributable to active cotransporter), thus increasing $C_{i}$, and negative during episodes when chloride flux is dominated by the synaptic current. $C$, Time course of $V$ and $d$ during an episode, revealing fast cycling; $d$ represents the fraction of nondepressed synapses. $\tau_{d} / \tau_{v}=4, g_{\text {syn }}=33 \mathrm{nS}$. Numbers and arrows on time courses refer to features of the episodic rhythm (see Results, Episodic behavior in the model and Fig. 3).

depolarized (Fig. 2A) and $\mathrm{Cl}^{-}$efflux is very strong (through synaptic channels), as indicated by negative values of $\mathrm{Cl}^{-}$flux in Figure $2 \mathrm{~B}$. As a consequence, $\mathrm{Cl}_{i}$ decreases (also shown in Fig. $2 B$ ), and eventually the driving force for synaptic current, $V-E_{\mathrm{Cl}}$, is not sufficiently excitatory to maintain activity, thereby ending an active phase. When $V-E_{C l}$ reaches its minimum, $\mathrm{Cl}^{-}$flux reverses, becoming positive, and slowly restores higher levels of $\mathrm{Cl}_{i}$. In this model, the instantaneous firing rate $f(V)$ can be small for low $V$ but never zero (and similarly for the synaptic current). Therefore, a certain fraction of cells is always spontaneously active, and this feature provides the opportunity for recurrent connections to build and propagate activity and generate a new episode.

Variations of $E_{\mathrm{Cl}}$ follow changes in the intracellular $\mathrm{Cl}^{-}$concentration (according to the Nernst relationship; Eq. 5), rising slowly during silent phases and decreasing faster during episodes. $E_{\mathrm{Cl}}$ spans a depolarized range (between -35 and $-27 \mathrm{mV}$ for this particular case; values for the parameters of the model are given in the figure legend), in good agreement with values deduced from experiments (Chub and O'Donovan, 2001). The model predicts that, during the time course of an episode and silent phase, 

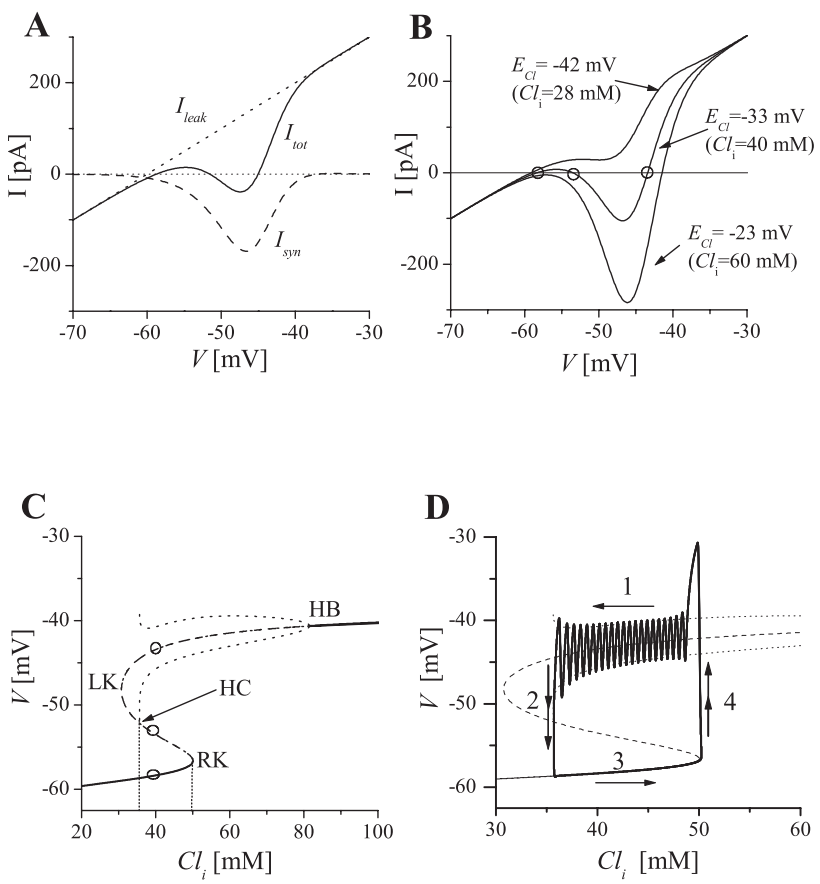

Figure 3. Emergence of bistability. $A$, Curves showing the variations with $V$ of $I_{\text {leak }}$ (dotted; outward for $V>V_{\text {rest }}$ ), steady-state $I_{\text {syn }}$ (dashed; inward, $E_{\mathrm{Cl}}=-37 \mathrm{mV}$ ), and $I_{\text {tot }}=I_{\text {leak }}+$ $I_{\text {syn }}$ (solid; N-shaped). $\boldsymbol{B}$, Current-voltage relationship $I_{\text {tot }}(\eta)$ for different $E_{\mathrm{Cl}}$ values. Steady states are defined by $I_{\text {tot }}(V)=0$ and thus graphically correspond to the intersections of the $I_{\text {tot }}(\eta)$ curve and the horizontal axis. When $E_{C I}$ is low (top curve), there is only one intersection, corresponding to a stable steady state of low voltage $\left(V\right.$ close to $V_{\text {rest }}$ ). When $E_{C \mid}$ is high (bottom curve), there is only one intersection, at high $V$. For intermediate values of $E_{\mathrm{C},}$ there are three intersections with the abscissa (open circles), corresponding to three steady states of different stability (see Results). C, Plot of the steady-state values of $V$ for a range of $C_{i}$ values. The resulting curve is $S$-shaped, with a lower branch, an upper branch, and a middle branch delimited by the left and right knees (LK and RK). The three steady states shown in $\boldsymbol{B}$ for $E_{\mathrm{Cl}}=-37$ $\mathrm{mV}$ are shown as open circles. Solid portions of the $S$-shaped curve (lower branch and part of the upper branch) indicate stable states; dashed portions (middle branch and part of the upper branch) indicate unstable states. At the transition from stable to unstable state on the upper branch (HB point), the $\mathrm{V}-\mathrm{d}$ system with $\mathrm{Cl}_{i}$ treated as a parameter becomes oscillatory, with minimum and maximum values of the voltage indicated by the dotted line. Cycling amplitude increases with decreasing $C l_{i}$ until the minimum of the oscillation intersects the middle branch (HC point). The $V-d$ subsystem is bistable for the $C_{i}$ range defined by values associated with $H C$ and RK. D, When $\mathrm{Cl}_{i}$ varies slowly according to Equation 3, it sweeps back and forth over the range of bistability, with the $V-d$ subsystem showing slow evolution (of cycling) during an episode with $\mathrm{Cl}_{i}$ decreasing and then increasing during the interepisode phase; rapid switching between the low activity state and the upper state of cycling occurs at the transition points $\mathrm{HC}$ and RK (see Results and Fig. $4 B$ ). The $V$ and $C I_{i}$ variables corresponding to the rhythmic time course in Figure 2 are projected here as a trajectory that falls precisely on the path that is predicted. The numbers and arrows here correspond to those from Figure 2 and they represent the following: 1, episode; 2, termination of episode and rapid switch to silent phase; 3 , the interepisode phase of low activity; 4, the rapid transition to start of next episode. $\tau_{d} / \tau_{v}=4 ; g_{\text {syn }}=33 \mathrm{nS}$.

the short-time averaged membrane potential $V$ is below $E_{\mathrm{Cl}}$ (and above $V_{\text {rest }}$ ). Examining the time courses within an episode (Fig. $2 C)$, we see the cycling activity of $V$, also in agreement with experimental traces (compare with Fig. $1 B$ ). The fast depression variable $d$ oscillates $\sim 90^{\circ}$ out of phase with $V$, peaking during the down stroke of $V$ during each cycle (Fig. 2C, bottom).

Insight into how the activity switches, between a low-voltage resting state and a high-voltage cycling one, emerges from examining the current-voltage ( $I-V$ ) relationship (Fig. 3 ). For this, we rely on the fact that the variable $C l_{i}$ varies at a much slower rate than $V$ and $d$. Therefore, at a given instant of time, we can think of $\mathrm{Cl}_{i}$ having a fixed value, and, to a first approximation, regard it as a parameter of the model rather than as a dynamic variable. We can then find the steady states of the system (that satisfy $\dot{V}=0$ with $d$ simultaneously satisfying $\dot{d}=0$ ) over a certain range of $C l_{i}$. Figure 3 shows a plot of the two current terms that contribute to voltage dynamics. The term $I_{\text {leak }}=g_{\text {leak }}\left(V-V_{\text {leak }}\right)$ is linear in $V$. Conversely, synaptic current $I_{\text {syn }}$ is non-zero only between $V_{\text {rest }}$ and $E_{\mathrm{Cl}}$ and has negative values because it is an inward current (carrying negatively charged $\mathrm{Cl}^{-}$ions outward). Here, we assume that the fast depression is equilibrated, i.e., we set $d=$ $d_{\infty}(V)$, and note that the slow variable $E_{\mathrm{Cl}}$ is fixed. The total current $I_{\text {tot }}=I_{\text {leak }}+I_{\text {syn }}$ gives rise to a curve whose shape depends on the $E_{\mathrm{Cl}}$ value (Fig. $3 B$ ). This value in turn affects the intersections that the curve has with the abscissa $(I=0)$. The voltage values for these zero-current crossings define the steady states of the system. In the case of low $E_{\mathrm{Cl}}\left(E_{\mathrm{Cl}}=-42 \mathrm{mV}\right.$ in the figure), there is only one intersection at low $V$ (at approximately -60 $\mathrm{mV}$ ). Here, the network is not very active because of its low functional connectivity [as implied by the sigmoidal shape of $f(V)$ ]. Conversely, if $E_{\mathrm{Cl}}$ is high enough $\left(E_{\mathrm{Cl}}=-23 \mathrm{mV}\right.$ in the figure), there is only one intersection at high $V$ (at approximately $-40 \mathrm{mV}$ ), corresponding to a state of steady high firing. Finally, for intermediate values of $E_{\mathrm{Cl}}$, the $I-V$ curve has three zerocurrent crossings. The low-voltage steady state is stable, the middle state is unstable, and the stability of the high state depends on the relative timescales of $V$ and $d$, i.e., on the ratio $\tau_{d} / \tau_{v}$ (see below, Changing $\tau_{d} / \tau_{v}$ and the emergence of oscillatory behavior).

The dependence of the steady states of the system on $C l_{i}$, when it is treated as a parameter, are compactly described with a plot in the $\mathrm{V}-\mathrm{Cl}_{i}$ plane (Fig. $3 \mathrm{C}$ ). The $\mathrm{S}$-shaped curve represents the voltage values at steady state over a range of $\mathrm{Cl}_{i}$ values. The lower branch of this S-curve corresponds to the low- $V$ zero-current crossing in Figure $3 B$. The upper branch of the S-curve corresponds to the high- $V$ zero-current crossing. In the $C l_{i}$ range between the left and right knees (LK and RK), there are three steady states of the $V$ - $d$ fast subsystem, with the middle branch of the $\mathrm{S}$-curve corresponding to the middle zero-crossing in Figure $3 B$. A steady state is unstable at points on the $\mathrm{S}$-curve that are dashed; solid denotes stable. Note that, over much of the $\mathrm{Cl}_{i}$ range shown here, the high- $V$ state is unstable. Hence, stability is not necessarily guaranteed, although the slope of the steady-state relationship, $I_{\text {tot }}$ versus $V$, is positive. The dynamic interaction between $V$ and $d$ affect the stability of the steady state. However, over much of the $\mathrm{Cl}_{i}$ range shown, here the high- $V$ steady state is "surrounded" by a stable high- $V$ oscillatory state, indicated by the dotted "fork" whose two branches correspond to the maximum and minimum $V$ values of the oscillation. These oscillations correspond to the cycling observed during an episode in the chick spinal cord. The high- $V$ oscillations begin at the point labeled HB (Hopf bifurcation), at which the steady state loses stability. As $\mathrm{Cl}_{i}$ decreases, the oscillation amplitude (peak to trough) grows. The oscillation disappears at the point labeled HC (homoclinic) when its trough decreases to just meet the middle- $\mathrm{V}$ value, when the minimum activity in a cycle drops to/below a threshold value.

A significant feature in this model is that, over the $\mathrm{Cl}_{i}$ range between $\mathrm{HC}$ and RK, the $V$ - $d$ subsystem is bistable. There is a stable state of high- $V$ oscillations (for cycling within an episode) and a coexistent stable low- $V$ steady state (that corresponds to the silent phase between episodes). One can imagine that, if $\mathrm{Cl}_{i}$ (treated as a control parameter here) were varied slowly backand-forth throughout this range (and just a bit beyond the two knees), that $V$ would exhibit the time course of an episodic rhythm, switching alternately between a silent phase and an ac- 
tive phase of cycling. This is the essence of what happens in the full three-variable model when $C l_{i}$, coupled mutually through $E_{\mathrm{Cl}}$ to the $V$ - $d$ dynamics, follows its dynamical prescription. To illustrate this point, we take the time course of $V$ and $C l_{i}$ (from Fig. 2) and project it as a trajectory (Fig. 3D) onto the (S with fork) diagram from Figure $3 C$. The numbers and arrows here correspond to those in Figure 2. During an episode (Fig. 3D, 1), the system is in the high- $V$ state of cycling; chloride channels are open, $\mathrm{Cl}^{-}$efflux dominates, and $\mathrm{Cl}_{i}$ slowly decreases. The system point is drifting leftward along the trajectory. When $\mathrm{Cl}_{i}$ decreases to a critical level (at $\mathrm{HC}$ ) and activity $V$ during a cycle falls below a threshold level, the episode terminates (Fig. 3D, 2) and $V$ drops down abruptly (Fig. $2 D$, double arrow) to a low value. During a silent phase (Fig. $3 D, 3$ ), when $I_{\text {syn }}$ is very small and the influx of the chloride cotransporter dominates, $\mathrm{Cl}_{i}$ slowly increases and, on the phase plane, the system drifts rightward along the lower branch of the S-curve. At a critical value of $\mathrm{Cl}_{i}$ (at $\mathrm{RK}$ on the $\mathrm{S}$-shaped curve), the low- $V$ steady state meets the slowly falling threshold (middle branch) and disappears; $V$ increases abruptly (Fig. 3D, 4), and a new episode begins.

Such bistability in the fast subsystem is a qualitative feature, also seen in our previous models for episodic rhythmogenesis (Tabak et al., 2000, 2001). Based on this bistability, we predicted and confirmed (experimentally and computationally) that, by using a brief stimulus, one could reset the rhythm, e.g., kick the system during the silent phase and abruptly into an episode. Moreover, we confirmed the additional prediction that the duration of the ensuing episode would be shorter if the kick occurred earlier in the silent phase (Tabak et al., 2001) .

Note that, during the silent phase, while tracking the lower branch, the system approximately satisfies $d V / d t=0$. The net ionic current is zero, and the voltage is essentially satisfying the equilibrium condition (Goldman-Hodgkin-Katz): $V \approx\left(g_{\text {leak }}\right.$ $\left.V_{\text {rest }}+g_{\text {syn,eff }} E_{\mathrm{Cl}}\right) /\left(g_{\text {leak }}+g_{\text {syn,eff }}\right)$, where $g_{\text {syn,eff }}=\mathrm{g}_{\text {syn }}{ }^{-}$ $d f(V)$. However, the system is slowly depolarizing. This is because $E_{\mathrm{Cl}}$ is slowly increasing as $\mathrm{Cl}_{i}$ increases. The slow depolarization is seen experimentally in many intracellular recordings.

As technical points, we note that response diagrams such as in Figure $3 C$ are called bifurcation diagrams; they indicate how features of solutions vary with parameters and critical parameter values in which solutions emerge or bifurcate. The high- $V$ oscillatory state emerges at point HB, via a Hopf bifurcation (Strogatz, 1994) (see also Rinzel and Ermentrout, 1998), in which the high- $V$ steady state undergoes an exchange of stability, changing from a damped to a growing oscillation as $\mathrm{Cl}_{i}$ decreases. The high- $V$ oscillatory state terminates at $\mathrm{HC}$ when the minimum $V$ just reaches the middle- $V$ steady state, forming a homoclinic orbit connection (Strogatz, 1994) (see also Rinzel and Ermentrout, 1998); at this point, the oscillation has zero frequency or infinite period. In our models for episodic rhythms, the cycle frequency drops precipitously at the end of an episode, as apparent in many experimental recordings.

\section{Changing $\tau_{d} / \tau_{v}$ and the emergence of oscillatory behavior}

As mentioned in Materials and Methods, the relative values of the time constants $\tau_{d}$ and $\tau_{V}$ significantly affect certain features of the model, especially whether or not bistability and cycling-like oscillations occur in the fast subsystem. Figure 4 illustrates how variations of the ratio $\tau_{d} / \tau_{v}$ lead to different behaviors in the full three-variable model and how these are understood from corresponding effects in the $V$ - $d$ subsystem. The left panels show the time courses of $V$ as obtained from simulations of the full system. The right panels, analogous to Figure $3 C$, show the response di-
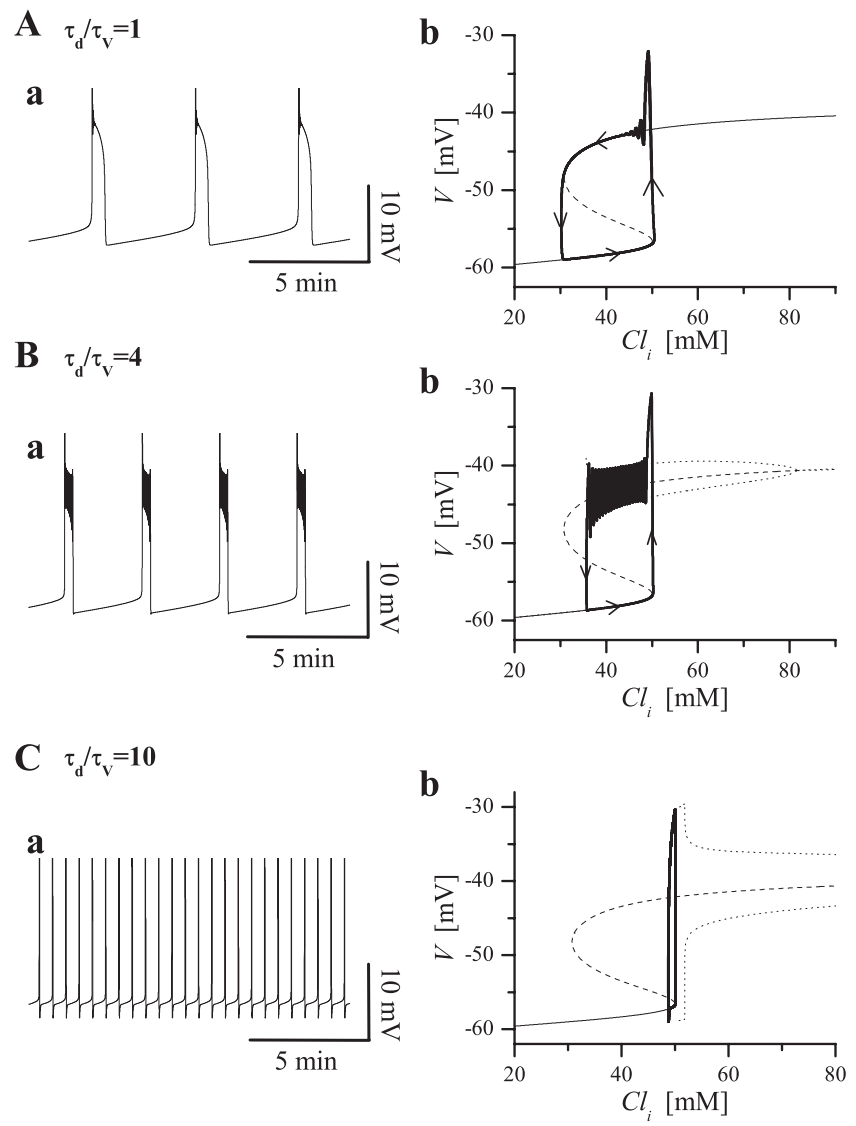

Figure 4. Dependence of bistable behavior on $\tau_{d} / \tau_{v}$ value. $A$, For low $\tau_{d} / \tau_{v}$ values $\left(\tau_{d} / \tau_{v}=\right.$ $1)$, the system is bistable but shows no oscillations in the upper state, as can be seen in the $V$ trajectory $(\boldsymbol{a})$ and in the response diagram $(\boldsymbol{b}) . \boldsymbol{b}$ also shows the trajectory on the $\mathrm{V}-\mathrm{Cl}_{i}$ plane, and arrows indicate the direction along which the system moves. $\boldsymbol{B}$, For $\tau_{d} / \tau_{v}=4$, the system is bistable, with a quiescent low-voltage state and an upper oscillatory state, as seen in the $V$ trajectory $(\boldsymbol{a})$ and in the response diagram $(\boldsymbol{b})$. During the silent phase, $\mathrm{Cl}_{i}$ slowly increases, and the system moves along the lower branch until it hits a threshold value (corresponding to the RK) and abruptly goes to the upper state. In the active phase, $\mathrm{Cl}_{i}$ decreases along the upper branch, in which it undergoes oscillations because of the presence of the fast depression variable $d$. When $\mathrm{Cl}_{i}$ reaches the low critical value (at the $\mathrm{HC}$ ), the voltage drops, and the system goes to the low quiescent state. $C$, For high $\tau_{d} / \tau_{v}$ values $\left(\tau_{d} / \tau_{v}=10\right)$, the system loses bistability and goes into continuous "spiking" [V trajectory $(\boldsymbol{a})$ and the response diagram $(\boldsymbol{b})$ ]

agrams on the $V-C l_{i}$ plane, i.e., the $S$-shaped curve. The trajectory of the full system is superimposed on the $V-C l_{i}$ plane, and, as we expect, it tracks along the stable states, circulating counterclockwise. Notice that, because different values of $\tau_{d} / \tau_{v}$ do not effect the values of steady states in the $V$ - $d$ subsystem, the shape of the S-shaped curve does not change with $\tau_{d} / \tau_{v}$, although the stability along the curve is strongly affected by the ratio $\tau_{d} / \tau_{v}$.

For low values of $\tau_{d} / \tau_{v}$ the negative feedback from depression is relatively fast. If it is too fast, cycling oscillations during the active phase are precluded, as seen in time course of $V$ (Fig. $4 A a$, for the case of $\left.\tau_{d} / \tau_{v}=1\right)$. The corresponding fast subsystem response diagram (Fig. $4 A b$ ) shows that the low- $V$ and high- $V$ steady states are each stable. Therefore, the system shows bistability over the full $\mathrm{Cl}_{i}$ range between LK and RK (Fig. $3 C$ ).

As $\tau_{d} / \tau_{v}$ is increased (Fig. $4 \mathrm{~B}$ ), the negative feedback caused by depression is delayed, and this leads to oscillations around the high- $V$ steady state (Fig. $4 \mathrm{Bb}$, dotted curves). The range of $C l_{i}$ for which the low-voltage steady state and the high-voltage oscillatory state coexist has changed with respect to the previous case. Now bistability in the fast subsystem occurs over a narrower $\mathrm{Cl}_{i}$ range, between the $\mathrm{HC}$ and RK (Fig. 3C). 
A $\tau_{\mathbf{d}} / \tau_{\mathbf{V}}=\mathbf{1}$
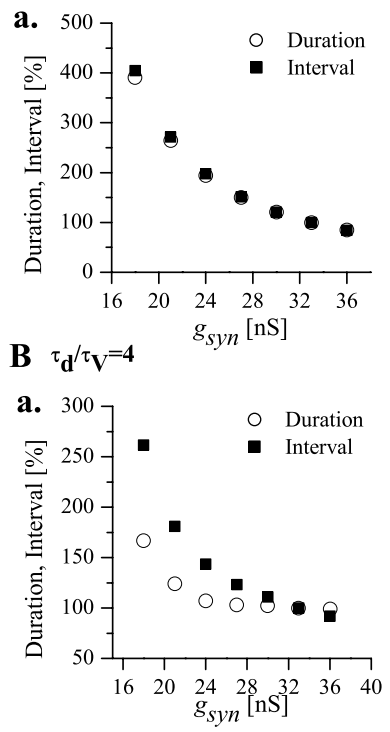

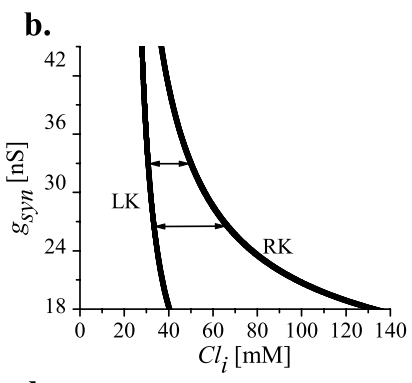

b.

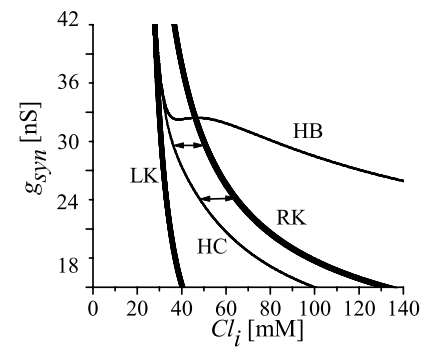

Figure 5. Dependence of interval and duration of episodes on $g_{\text {syn }}$ and $\tau_{d} / \tau_{v} \cdot A, \tau_{d} / \tau_{v}=1$. $\boldsymbol{a}$, Duration (open circles) and interval (filled squares) of episodes obtained from simulations for different $g_{\text {syn }}$ values. Data for duration (interval) is normalized to the duration (interval) value at $g_{\text {syn }}=33$ pS (control value, also used in Fig. 6). $\boldsymbol{b}$, Two-parameter response diagrams show how the right knee and the left knee shift with $g_{\text {syn. }}$. The bistability range (range of values covered by $\mathrm{Cl}_{i}$ during episodic behavior) increases with decreasing $g_{\text {syn }}$; consequently, both interval and duration increase with decreasing $g_{\text {syn }}$. Double arrows indicate the $C_{i}$ range covered during episodic activity, for two values of $g_{\text {syn }}$ ( 33 and $27 \mathrm{nS}$ ). $\boldsymbol{B}, \tau_{d} / \tau_{v}=4$. $\boldsymbol{a}$, Duration and interval for different $g_{\text {syn }}$ values. $\boldsymbol{b}$, Two-parameter response diagrams show how the RK and LK (thick lines) and the $\mathrm{HC}$ and $\mathrm{HB}$ (thin lines) shift with $g_{\text {syn }}$. Contrary to the previous case, $\mathrm{Cl}_{i}$ covers the range between $\mathrm{HC}$ and RK during episodic activity (double arrows; shown for $g_{\text {syn }}=$ 33 and $27 \mathrm{nS}$ ). With decreasing $g_{\text {syn }}$, this range does not increase as much as in $A$, so interval and durations are less affected.

As $\tau_{d} / \tau_{v}$ is further increased, the range of bistability is further decreased and disappears (Fig. $4 \mathrm{Cb}$ ), as $\mathrm{HC}$ moves to coincide with RK. In this case, the system goes into a regimen of continuous cycling (Fig. 4Ca); such oscillations might be viewed as single-cycle episodes.

In studying the model, we focused our attention on the range of $\tau_{d} / \tau_{v}$ values $(\sim 1-5)$ in which bistability in the $V$ - $d$ subsystem is expressed over a wide range of $g_{\text {syn }}$ values. With larger values of this ratio, the homoclinic point moves closer to the right knee as $g_{\text {syn }}$ is decreased, thereby reducing the range for bistability. The consequences of this are considered more fully in Discussion.

\section{Effects of $g_{\text {syn }}$ in the model}

Within this restricted range of $\tau_{d} / \tau_{v}$ values, we explored the dependence of the model on other parameters that can be related to experimentally accessible variables, thereby allowing for comparison between theory and experiments. First, we investigated how the model was affected by changing the synaptic conductance $g_{\text {syn }}$, anticipating experiments in which chloride synaptic conductance was decreased, i.e., experiments in which GABAergic conductances were partially blocked (see below, Effects of GABA antagonists in experiments).

Our simulations show that the episode duration and interepisode interval are affected by $g_{\text {syn }}$ in ways that differ with the $\tau_{d} / \tau_{v}$ ratio, shown in Figure 5 (left) for two values of $\tau_{d} / \tau_{v}$. Figure 5 (right) presents response diagrams for the $V$ - $d$ subsystem, which show how critical features of the S-shaped curve, including the $\mathrm{Cl}_{i}$ range for bistability of Figure $3 C$, shift when $g_{\text {syn }}$ is changed.
In the case of $\tau_{d} / \tau_{v}=1$ (Fig. $5 A a$ ), we found that both interval (filled squares) and duration (open circles) increased as $g_{\text {syn }}$ decreased. The two-parameter response diagram of Figure $5 A b$ defines the range of $g_{\text {syn }}$ and $C l_{i}$ values over which the bistable regimen occurs, i.e., the area between the right knee and the left knee curves obtained as the two parameters vary. When, during a silent phase, the system follows the low- $V$ branch of the S-shaped curve of Figure $4 A b, C l_{i}$ increases. This corresponds, in Figure $5 A b$, to moving from the LK curve toward the RK curve, at a fixed value of $g_{\text {syn }}$. Analogously, during an episode, the system moves leftward from the RK curve to the LK curve, covering the same $C l_{i}$ interval as it did during a silent phase but in the opposite direction.

If $g_{\text {syn }}$ is decreased, the system moves along another parallel line, lower than the preceding one (still between the two curves), and $\mathrm{Cl}_{i}$ is shifted toward higher values. This upward shift in the $\mathrm{Cl}_{i}$ range leads to an increase in both duration and interval when we decrease $g_{\text {syn }}$.

When $\tau_{d} / \tau_{v}$ is increased to 4 (Fig. $5 B$ ), interval and duration of episodes are affected differently than in the previous case. This is because now the $\mathrm{Cl}_{i}$ range spanned is determined by the interval between the right knee RK and the homoclinic point HC (Fig. $5 B b$ ) rather than the left knee as in the previous case. Simulations show that decreasing $g_{\text {syn }}$ over a certain range $(18-36 \mathrm{nS})$ leads to essentially no change in duration, whereas interval decreases significantly (Fig. $5 \mathrm{Ba}$, open circles and filled squares, respectively). The difference with the previous case $\left(\tau_{d} / \tau_{v}=1\right.$, no oscillations) is attributable to the fact that the oscillatory states are affected by a change in $g_{\text {syn }}$ in a different way than the steady states.

\section{Effects of GABA antagonists in experiments}

To reproduce experimental conditions comparable with the theoretical ones, we performed experiments in the presence of cholinergic blockers (50 $\mu \mathrm{M}$ mecamylamine, $50 \mu \mathrm{M} \mathrm{DH} \beta \mathrm{E}$, and $5 \mu \mathrm{M}$ atropine) and glutamatergic blockers ( $5 \mu \mathrm{M}$ CNQX and $50 \mu \mathrm{M}$ AP-5) to isolate the GABA/glycinergic network. This was regarded as our control condition. Our goal was then to reduce chloride conductance, a manipulation that corresponded to lowering $g_{\text {syn }}$ in the model. To do so, we bath applied low doses of GABAergic receptor antagonists: either the competitive $\mathrm{GABA}_{\mathrm{A}}$ antagonist bicuculline (1-2 $\mu \mathrm{M})$ or the neurosteroid DHEAS (5 $\mu \mathrm{M}$ ), an allosteric $\mathrm{GABA}_{\mathrm{A}}$ receptor modulator (Majewska et al., 1990; Shen et al., 1999) that reduces open-channel frequency (Mienville and Vicini, 1989). By using two different antagonists, we ensured that the effect was not dependent on the specific blocker.

We first tried to establish how much GABAergic transmission was reduced by the concentrations of bicuculline that we used. This was done by comparing the amplitude of ventral root potentials evoked by stimulation of the VLF before and after drug application. Because these experiments were performed in the presence of glutamatergic and cholinergic blockers, we assumed that the responses we were recording were predominantly GABA/ glycinergic. Also, because synaptic responses are depressed after an episode (Fedirchuk et al., 1999), we waited several minutes after an episode before applying the antagonist, thus ensuring that the evoked response had recovered. We then compared the averaged response taken after the antagonist application with the one right before the application, by measuring the amplitude within $5 \mathrm{~ms}$ of the onset, to include only the monosynaptic component (see Materials and Methods). We found that, on average, the response was decreased by $26 \pm 9 \%(n=4 ; p<0.05)$ by bicuculline (a similar decrease was obtained with DHEAS in two other experiments). This decrease can therefore be taken as an 


\section{EXPERIMENTS}

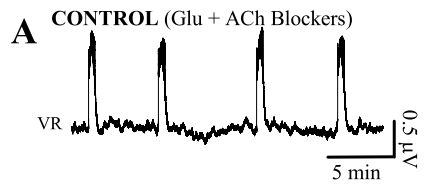

(Glu + ACh Blockers)+Bicuculline $2 \mu \mathbf{M}$

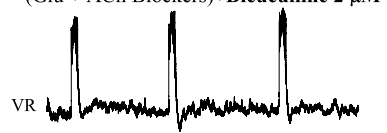

MODEL
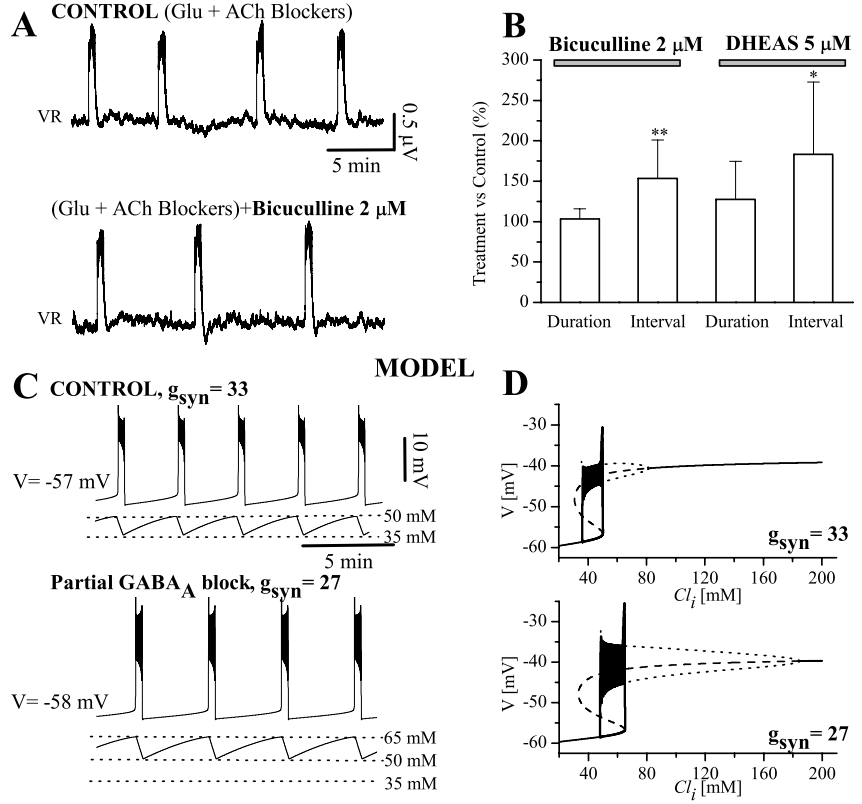

Figure 6. Effects of decreased synaptic transmission on episodic activity. $\boldsymbol{A}$, Ventral root recordings from an $\mathrm{E} 10$ embryo cord. Control condition is in the presence of glutamate (Glu) and acetylcholine $(A C h)$ receptor blockers. When bicuculline $(2 \mu \mathrm{m})$ is added, the interepisode interval increases (in this case, by $22 \%$ ), whereas duration shows no significant change. $\boldsymbol{B}$, Interval and duration of episodes for pooled data, in the case of bicuculline $(n=11)$ or DHEAS ( $n=$ 8). Data are normalized with respect to control. All experiments were performed in the presence of glutamatergic and cholinergic blockers. Only interval is significantly different from control $\left({ }^{*} p<0.05 ;{ }^{* *} p<0.005\right)$. C, Time course of $V$ and $C l_{i}$ from simulations $\left(\tau_{d} / \tau_{v}=4\right)$ for $g_{\text {syn }}=$ $33 \mathrm{nS}$ (corresponding to control) and $g_{\text {syn }}=27 \mathrm{nS}$ (corresponding to bicuculline treatment). Interval in this case increases by $19 \%$, and $\mathrm{Cl}_{i}$ range is shifted upward. $\boldsymbol{D}$, Response diagram and trajectory on the $\mathrm{V}-\mathrm{Cl}_{i}$ plane for $g_{\text {syn }}=33$ and $27 \mathrm{nS}$; the $\mathrm{Cl}_{i}$ range over which bistability occurs is higher for lower $g_{\text {syn }}$.

indication of the amount of reduction in chloride synaptic transmission in the network and can be used to make comparisons with $g_{\text {syn }}$ manipulations made in the model.

Having established the amount by which synaptic transmission was depressed by low doses of bicuculline $(1-2 \mu \mathrm{M})$ or DHEAS $(5 \mu \mathrm{M})$, we investigated the effects of such reduction in GABA transmission on the duration of active and silent phases. Application of the blocker usually stopped episodes for some time, prolonging the silent phase from tens of minutes to up to $1-2 \mathrm{~h}$. After this adjustment period, activity resumed at a slower rate.

Figure $6 A$ illustrates the slowing of spontaneous episodes by a low dose of bicuculline, and Figure $6 B$ summarizes results for interval and duration with the two treatments. We found that episode duration was not significantly affected in either case, whereas the interepisode interval significantly increased. In the presence of bicuculline, the interepisode interval lengthened to $166 \pm 50 \%(n=11 ; p<0.005)$ of control, whereas for DHEAS the increase was $180 \pm 80 \%(n=8 ; p<0.05)$. Episode duration was similar to control in the presence of either drug (bicuculline, $104 \pm 12 \%, n=11, p>0.05$; DHEAS, $129 \pm 44 \%, n=$ $8, p>0.05)$.

\section{Constraining model parameters with experiments on synaptic blockade}

We found experimentally that inhibiting chloride currents leads to a lengthening of the silent phase, although the active phase duration is not significantly affected. Our model best predicted this behavior in the case of $\tau_{d} / \tau_{v}=4$ (Fig. $5 \mathrm{Ba}$ ). In fact, for a change of $g_{\text {syn }}$ from 33 to $27 \mathrm{nS}$, there is good agreement between theory and experiments, in which an increase in interval and no significant change in episode duration are obtained (Fig. 6C).

In the model, the correct change in interval is given by a conductance change of $\sim 20 \%$. This is in accordance with the amount of depression of the synaptic potential that we obtained experimentally from the evoked responses $(26 \pm 9 \%$ in the case of bicuculline).

The prediction for the effects on system behavior when $g_{\text {syn }}$ is decreased can also be visualized, for the model, on the $\mathrm{V}-\mathrm{Cl}_{i}$ response diagram (Fig. $6 D$ ) (fixed ratio, $\tau_{d} / \tau_{v}=4$ ). In this case, the $V$ values for the lower and upper states are hardly changed, whereas the S-curves and cycling regimen (between $\mathrm{HC}$ and $\mathrm{HB}$ points) shift rightward by different amounts. This leads to a higher operating range of $C l_{i}$, as well as to a change in amplitude of the oscillations. In particular, for lower $g_{\text {syn }}$, the $\mathrm{Cl}_{i}$ range spanned is at higher values (Fig. 6C,D). Simulations show that, for this value of $\tau_{d} / \tau_{v}$, the duration of episodes is not significantly affected, whereas the interepisode interval is lengthened, as shown in the simulation of Figure 6C.

\section{Effects of changing $\mathrm{Cl}^{-}$flux dynamics}

Additional comparison between model and experiments comes from analyzing the response of the system to changes in parameters related to the $\mathrm{Cl}^{-}$flux, such as the exchange rate of the cotransporter and $\left[\mathrm{Cl}^{-}\right]_{\text {ext }}$.

Experiments to influence these two sources of $\mathrm{Cl}^{-}$flux are presented in Figure 7. First, we tested the effects of applying bumetanide, a blocker of the cotransporter. If the $\mathrm{Cl}^{-}$dynamics regulate activity as we suggest, pharmacological blockade of the cotransporter with bumetanide (under glutamatergic blockade) should abolish activity. We found that even low doses $(10 \mu \mathrm{M})$ of the blocker completely and reversibly abolished spontaneous activity (Fig. 7A, top). We found (Fig. 7A, bottom) that, in our experimental condition, bumetanide only slightly reduces the GABAa conductance from $2.9 \pm 0.5$ to $2.6 \pm 0.4 \mathrm{nS}$, although the change was not statistically significant ( $n=4 ; t$ test).

Another way to affect $\mathrm{Cl}^{-}$flux, and therefore $\mathrm{Cl}_{i}$ dynamics (in both experiments and simulations), is to manipulate $\left[\mathrm{Cl}^{-}\right]_{\text {ext }}$. We anticipated that such manipulations would affect not only the chloride equilibrium potential but also the rate of inward chloride pumping $\left(R_{\text {co-trans }}\right)$. Changing $\left[\mathrm{Cl}^{-}\right]_{\text {ext }}$ modifies the NKCC1 rate (Russell, 2000), although the precise quantitative relationship between the two variables is unknown for chick spinal neurons. Experimentally, we found that decreasing $\left[\mathrm{Cl}^{-}\right]_{\text {ext }}$ led to a significant increase in the interepisode interval, with little effect on episode duration (Fig. $7 B$ ).

To dissociate the effects of changes in $E_{\mathrm{Cl}}$ and $R_{\text {co-trans }}$ on episode behavior, we performed model simulations in which changes in extracellular chloride produced either a change in $E_{\mathrm{Cl}}$ or alternatively altered both $E_{\mathrm{Cl}}$ and $R_{\text {co-trans }}$. We first ran simulations in which changes of $\left[\mathrm{Cl}^{-}\right]_{\text {ext }}$ only affected $E_{\mathrm{Cl}}$. When $\left[\mathrm{Cl}^{-}\right]_{\text {ext }}$ was decreased, the duration of both the episode and the interepisode interval decreased. Conversely, when $\left[\mathrm{Cl}^{-}\right]_{\text {ext }}$ was increased, both phases lengthened (Fig. 8A). To understand this, consider a sudden reduction of extracellular chloride during the interepisode interval. This will elevate $E_{\mathrm{Cl}}$ and the driving force for GABA/glycinergic synaptic potentials which, in turn, will produce a premature episode. Because the interepisode interval is shortened, intracellular chloride will not recover to its usual preepisode level. In addition, this premature episode will be slightly longer than normal, resulting in a greater efflux of chloride. As a 
A
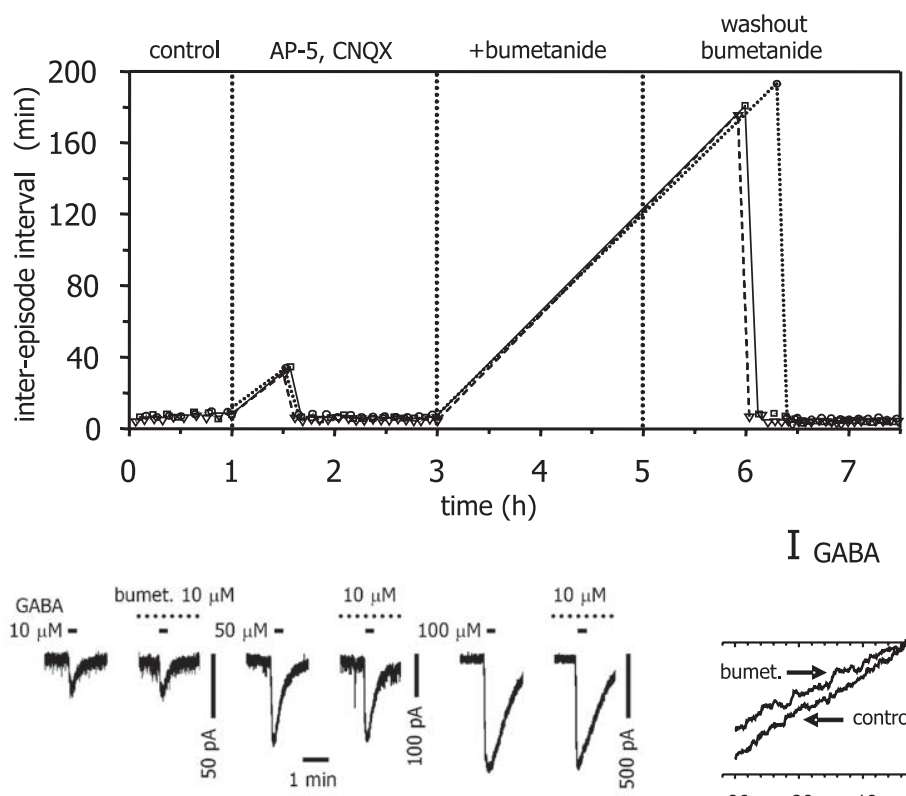

control

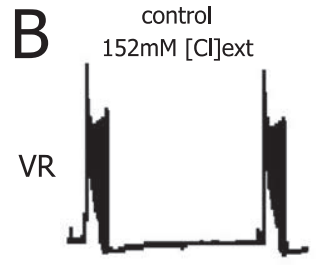

$137 \mathrm{mM}[\mathrm{Cl}] \mathrm{ext}$
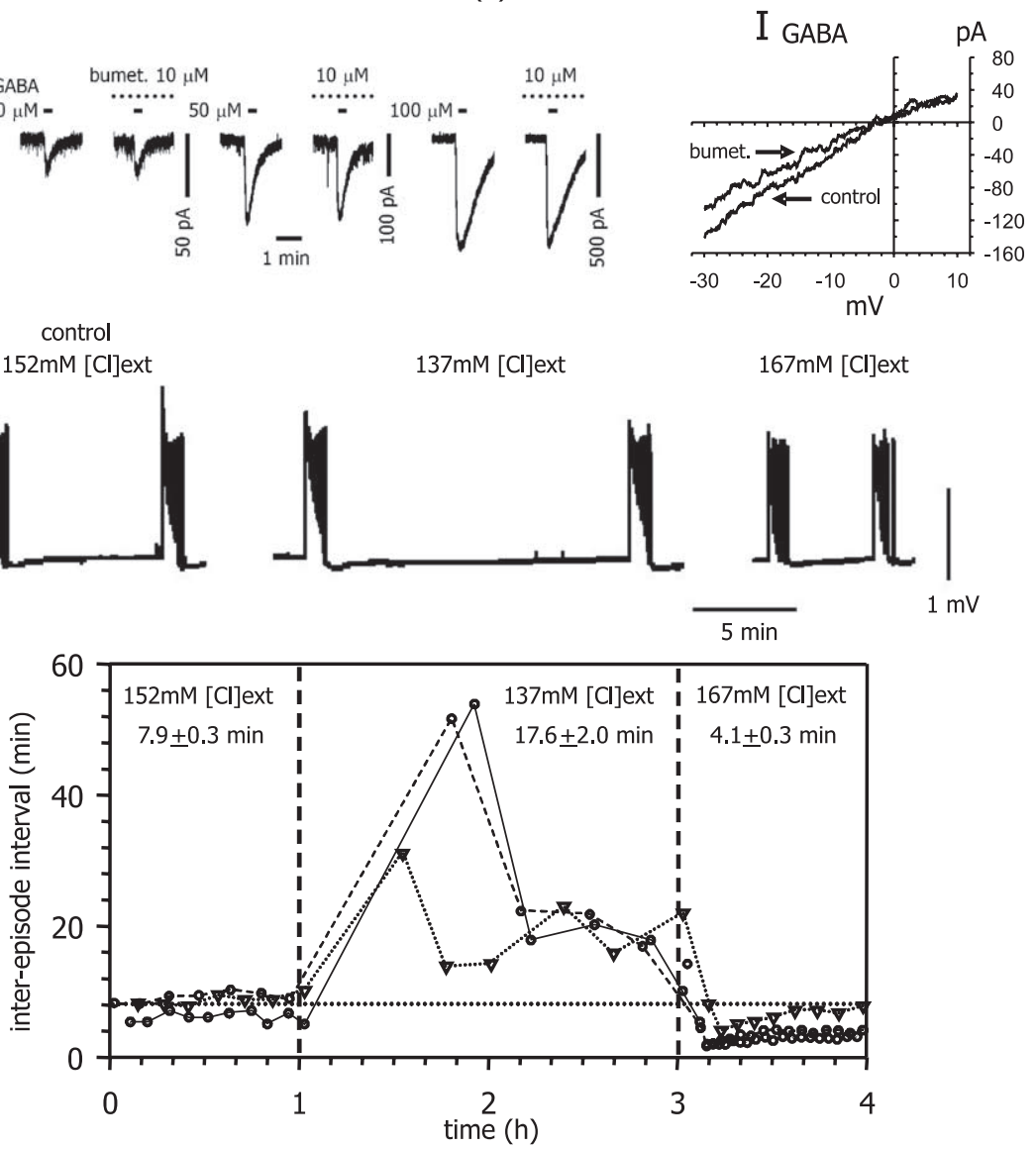

Figure 7. Experimental manipulations of $\mathrm{Cl}^{-}$flux. $\boldsymbol{A}, \mathrm{Blockade}$ of the $\mathrm{Cl}^{-}$cotransporter with bumetanide. Top, Interepisode intervals before, during ( $50 \mu \mathrm{m}$ AP-5 plus $20 \mu \mathrm{m}$ CNQX) plus $10 \mu \mathrm{m}$ bumetanide, and during washout of bumetanide. Bumetanide (bumet) completely suppressed activity, which recovered after an initial blockade ( 3 experiments, in the presence of AP-5 and CNQX, E10-E11 embryos). Bottom, Left, Whole-cell current response of spinal neuron to short (20 s) pressure applications of different $\mathrm{GABA}$ concentrations (10,50, and $100 \mu \mathrm{m}$; marked by short lines) in the absence or presence of bath-applied bumetanide (10 $\mu \mathrm{m}$; marked by dotted lines). Bumetanide does not block the GABAergic current. Currents were recorded from the same cell at a holding potential of $-40 \mathrm{mV}$. Current-voltage relationship for $50 \mu \mathrm{m} \mathrm{GABA}$ application, under control conditions, and in the presence of $10 \mu \mathrm{m}$ bumetanide (shown on right). Bumetanide has no effect on the reversal potential for GABA but has a slight effect on the conductance. GABA and bumetanide were applied as in the left panel experiments, but a voltage-ramp protocol was used for measurement of the current-voltage relationship. $\boldsymbol{B}$, Manipulations of $\left[\mathrm{Cl}^{-}\right]_{\text {ext }}$. Top, Ventral root recordings for three different values of $\left[\mathrm{Cl}^{-}\right]_{\text {ext }}$. Decreasing the $\left[\mathrm{Cl}^{-}\right]_{\text {ext }}$ has minimal effect on episode duration but strongly increases interepisode interval. Bottom, Plot of the interepisode intervals under three different $\left[\mathrm{Cl}^{-}\right]_{\text {ext }}$ (as in the top panel in $\boldsymbol{B} ; 3$ experiments). Numbers on graph are mean $\pm \mathrm{SE}$, which at $p=0.05$ were significantly different (ANOVA test). The changes in extracellular chloride were made in the presence of glutamatergic antagonists (for additional details, see Materials and Methods).

consequence, the system will now operate at a lower intracellular chloride concentration, and $E_{\mathrm{Cl}}$ will be to close to its value before the external chloride was changed. A similar argument applies to increases of $\left[\mathrm{Cl}^{-}\right]_{\mathrm{ext}}$.
$167 \mathrm{mM}[\mathrm{Cl}] \mathrm{ext}$

These results differ from those observed experimentally. To investigate this further, we performed simulations in which both $E_{\mathrm{Cl}}$ and $R_{\text {co-trans }}$ changed in response to external chloride (Fig. $8 \mathrm{~B}$ ). We allowed $R_{\text {co-trans }}$ to change by $\pm 11 \%$ for a correspondent increase or decrease of 15 $\mathrm{mM}$ in external chloride. When external chloride was reduced, the interepisode interval lengthened (Fig. 8B), consistent with the experimental data. Accordingly, when it was increased, the interepisode interval shortened. Although there were small changes in episode duration, the dominant effects were on the interepisode interval. These results suggest that the effects of changing extracellular chloride on episodic behavior are primarily attributable to its effects on inward chloride pumping (i.e., on $R_{\text {co-trans }}$ ).

Why do changes in $R_{\text {co-trans }}$ have such powerful effects? This parameter directly affects the net $\mathrm{Cl}^{-}$flux. These effects can be more easily understood using another graphical representation of the model. We use a response diagram analogous to that of Figure $3 C$, but now, instead of $V$, we plot as a measure of response the $\mathrm{Cl}^{-}$flux (per unit volume) through synaptic channels versus $C l_{i}$. We let $J_{\text {syn }}=-I_{\text {syn }} /$ $\left(F \cdot \mathrm{Vol}_{\mathrm{Cl}}\right)$ (flux per volume) and plot its value given the steady-state values of $V$ and $d$ (i.e., that correspond to $\dot{V}=0, \dot{d}=0$ ) versus $\mathrm{Cl}_{i}$ to get an S-shaped curve (Fig. 9). We also plot the $\mathrm{Cl}^{-}$flux per volume through the cotransporter, $J_{\text {co-trans }}=$ $R_{\text {co-trans }} / \mathrm{Vol}_{\mathrm{Cl}}$ (horizontal line). Now to analyze the dependence on $R_{\text {co-trans }}$, we refer back to the $\mathrm{Cl}^{-}$balance equation, Equation 3 in Materials and Methods, which can be rewritten as $d C l_{i} / d t=J_{\text {co-trans }}$ $-J_{\text {syn. }}$. If $J_{\text {co-trans }}>J_{\text {syn }}, C l_{i}$ is increasing, whereas it is decreasing if the inequality is opposite. When we compare the curve for $J_{\text {syn }}$ and the horizontal line corresponding to the constant $J_{\text {co-trans, }}$, we see graphically that $\mathrm{Cl}_{i}$ increases during the silent phase and decreases during the active phase. Moreover, we see now that, if $R_{\text {co-trans }}$ is decreased (the horizontal line drops down), the velocity of $\mathrm{Cl}_{i}$ is decreased in the silent phase and increased in the active phase, i.e., the interepisode interval increases as we expect intuitively. This treatment predicts that, as $R_{\text {co-trans }}$ is decreased, the percentage of active phase decreases and also that the $\mathrm{Cl}_{i}$ range during the episodic pattern is independent of $R_{\text {co-trans }}$. In addition, if $R_{\text {co-trans }}$ is decreased such that the horizontal line representing $J_{\text {co-trans }}$ intersects the lower branch of the S-shaped $J_{\text {syn }}$ curve (Fig. 9, dotted horizontal line), activity stops because there is no net $\mathrm{Cl}^{-}$flux to switch the network to the active phase. It will be worthwhile to test these theo- 
A
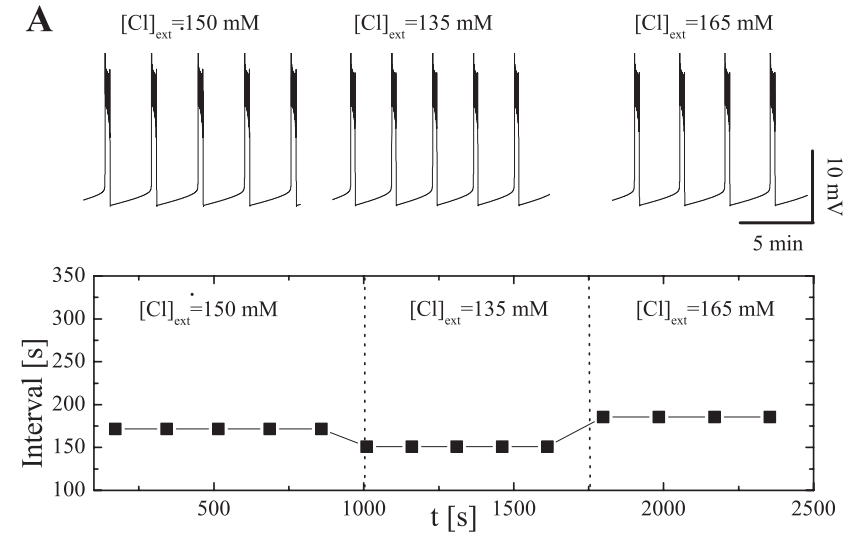

B

$$
\begin{array}{lll}
R=110^{-8} & R=0.88510^{-8} & R=1.11510^{-8} \\
{[\mathrm{Cl}]_{\mathrm{ext}}=150 \mathrm{mM}} & {[\mathrm{Cl}]_{\mathrm{ext}}=135 \mathrm{mM}} & {[\mathrm{Cl}]_{\mathrm{ext}}=165 \mathrm{~m}}
\end{array}
$$
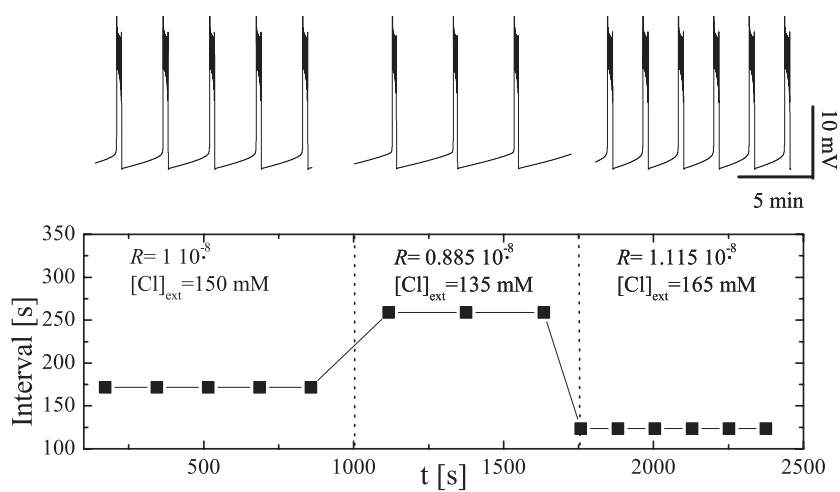

Figure 8. Model behavior under different external $\mathrm{Cl}^{-}$concentrations. $\boldsymbol{A}$, Variations of $\left[\mathrm{Cl}^{-}\right]_{\mathrm{ext}}$. Top, Time course of average membrane potential under diferent conditions. $\left[\mathrm{Cl}^{-}\right]_{\mathrm{ext}}$ minimally affects episode duration and interepisode interval. Bottom, Plot of the interval under the same conditions as in the top panel. Compare with Figure $7 B$. B, Variations of $\left[\mathrm{Cl}^{-}\right]_{\text {ext }}$ and $R_{\text {co-trans }}$ (rate of $\mathrm{Cl}^{-}$cotransport). $R_{\text {co-trans }}$ is changed in parallel with $\left[\mathrm{Cl}^{-}\right]_{\text {ext }}$ Top, Time course of average membrane potential under different conditions. Decreasing $R_{\text {co-trans }}$ increases interepisode interval. Bottom, Plot of the interval under the same conditions as in the top panel. Results are similar to experimental results shown in Figure $7 B$.

retical predictions when a selective blocker of the $\mathrm{Cl}^{-}$cotransporter that allows for titrating its rate becomes available.

\section{Discussion}

We formulated and implemented a new firing rate model (i.e., mean $V$ ) to help explain the emergence of spontaneous rhythmic activity in the developing chick spinal cord. Our model shows how a biophysical mechanism based on chloride dynamics (i.e., slow variations in $\mathrm{Cl}^{-}$concentration) can underlie the episodic rhythmic activity. It involves three essential mechanistic ingredients: bistability in the recurrent (functionally) excitatory network, constraints on the timescales $\tau_{d}$ and $\tau_{v}$ (to achieve cycling during episodes), and slow dynamics of $\mathrm{Cl}^{-}$handling. Admittedly, our mean field description is highly idealized compared with a biophysically detailed cell-based network model. Nevertheless, our model incorporates plausible chloride dynamics. Values for the chloride conductance, the leak conductance, and the rate of inward chloride pumping are in fact derived from measurements on embryonic chick spinal neurons (Chub and O'Donovan, 2001). However, for the emergence of bistability, it is the ratio of conductances (total synaptic to leakage) that is more critical than the absolute value of these parameters. Episodic activity in the model occurred only when the ratio was large enough but within a fairly broad range of values $(\sim 6-12)$.

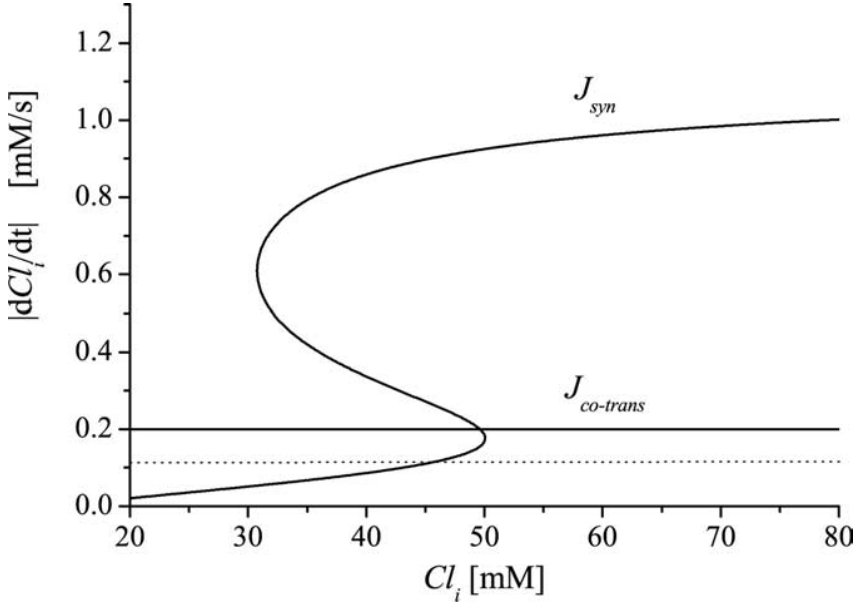

Figure 9. Response diagram for $J_{\text {syn }}=-I_{\text {syn }} /\left(F \cdot V 0 I_{C l}\right)$. This diagram shows the steady-state $\mathrm{Cl}^{-}$flux per volume attributable to synaptic current $\left(J_{\text {syn }} ;\right.$ solid $S$-shaped curve) and the opposite of the $\mathrm{Cl}^{-}$flux per volume attributable to the $\mathrm{Cl}^{-}$cotransporter ( $J_{\text {co-trans }}$; horizontal line). It is similar to the $V$ response diagram but allows graphical determination of the net $\mathrm{Cl}^{-}$flux. The derivative of $C I_{i}$ is equal to $\left(J_{\text {co-trans }}-J_{\text {syn }}\right)$ and therefore is represented on the diagram by the vertical distance between the horizontal line and the $\mathrm{S}$-shaped curve. During the interepisode interval, the system is tracking the lower branch, so the derivative is small but positive ( $\mathrm{Cl}^{-}$slowly flows inside). During an episode, the system tracks the upper branch, and the derivative is large and negative (large outward $\mathrm{Cl}^{-}$flux). If the cotransporter rate is decreased, the horizontal line moves down, closer to the lower branch. This decreases the inward $\mathrm{Cl}^{-}$flux during the intervals and (slightly) increases the outward $\mathrm{Cl}^{-}$flux during the episodes. If $\mathrm{s}_{\text {co-trans }}$ is too small (dotted horizontal line), the horizontal line moves under the right knee, its intersection with the lower branch of the $\mathrm{S}$-shaped curve defines a stable steady state, and episodic activity is lost.

\section{Time constants of the model}

There are two distinct timescales in the model: a slow one, determined by $\mathrm{Cl}^{-}$dynamics (giving rise to episodes), and a fast one, determined by $\tau_{d}$ and $\tau_{v}$ (underlying the cycling and its frequency). The parameter $\tau_{v}$, appearing in the voltage equation, is not simply a membrane time constant but also reflects the timing of the network input to the neuron. Therefore, it can be related to a "network recruitment time constant" that accounts for buildup of activity within the population of neurons. We estimated this value based on the time-to-peak of the ventral root potential recorded at episode onset. Setting $\tau_{v}$ in this manner resulted in intraepisode cycle frequencies that are in good agreement with those observed experimentally $(\sim 1 \mathrm{~Hz}$ at the beginning of the episode).

A critical parameter in the model is the ratio $\tau_{d} / \tau_{v}$. We found that this ratio had to be within a certain range for the model to display fast cycling. If it was too small, i.e., fast depression was speeded or network recruitment was slowed, cycling was lost (although bistability and cycle-less episodes were retained). If, conversely, $\tau_{d} / \tau_{v}$ was too big, i.e., depression is slow compared with network recruitment, then bistability was lost and the model did not exhibit episodes of activity. The sensitivity of our model to the ratio of these time constants is analogous to that of conductance-based models of bursting for neurons or pancreatic $\beta$-cells. In such models, the timescale in the fast subsystem of negative feedback and that of the autocatalytic process should be comparable (Rinzel, 1985; Sherman et al., 1988).

As the ratio $\tau_{d} / \tau_{v}$ increased, on the two parameter response diagrams (Fig. 5B) the homoclinic point moved toward the right knee, thereby limiting the range of bistability. Under these conditions, decreasing $g_{\text {syn }}$ led to an increase in interepisode interval and no significant change in the duration of the episode, as ob- 
served experimentally. Additional (but not too much) increase of the ratio led to a decreasing trend of both episode duration and interepisode interval (with decreasing $g_{\text {syn }}$ ), which was not observed experimentally. Increasing $\tau_{d} / \tau_{v}$ even more eliminated typical episodic behavior and led to single-cycle rhythmicity, a prediction for possible future experiments.

\section{Comparison of model predictions and experiment}

We found that a $20 \%$ decrease in $g_{\text {syn }}$ replicated an $\sim 25 \%$ decrease in the amplitude of GABAa/glycinergic ventral root potentials, for a reasonable range of $\tau_{d} / \tau_{v}$, as mentioned above. We also found that the effects of changing extracellular chloride on interepisode interval could be explained if we assumed that $R_{\text {co-trans }}$ covaried with the extracellular chloride concentration (Russell, 2000). We found, however, that the experimentally observed increase in interepisode interval in the presence of reduced extracellular chloride (Fig. 7B) differed from the model results (Fig. $8 B$ ) in two respects. First, the model did not exhibit the partial recovery shown by the experiment. Experimentally, we found that the interepisode interval initially increased by $\sim 3-5 \times$ when the external $\mathrm{Cl}^{-}$concentration was reduced and then recovered to a stable lower $(2 \times)$ level. The most likely explanation for this difference is that experimental changes in external chloride will affect other systems not represented in the model. In our previous model (Tabak et al., 2000, 2001), we note that spontaneous activity naturally compensates for externally imposed perturbations, and this may be another example of this homeostatic effect. The second difference between theory and experiment was that the experimental increase in the interepisode intervals was greater $(\sim 3-5 \times)$ than in the simulations $(\sim 1.5)$. The smaller changes in the interepisode intervals of the model probably occurred because the decrease in $R_{\text {co-trans }}$ was limited to $\sim 11 \%$. If it was lower than this, episodic activity ceased.

Our model gave several important results regarding $\mathrm{Cl}^{-} \mathrm{dy}-$ namics that could be tested in future experiments using chloridesensitive electrodes or imaging. First, in the model, the mean membrane potential $V$ is always lower than $E_{\mathrm{Cl}}$ throughout the episode, implying that GABA is depolarizing throughout the episode. Second, the model predicts an upward shift of the $C l_{i}$ range as $g_{\text {syn }}$ decreases. This mechanism acts to compensate the decrease in $g_{\text {syn }}$ (within certain limits) and allows episodes to recover without the involvement of any additional mechanisms. This prediction is analogous to the one that emerged in the previous $s$ model, in which a loss of glutamatergic synapses was compensated by an increase in the amplitude of GABA/glycinergic synaptic potentials (Tabak et al., 2001).

\section{Limitations of the model}

Our model is necessarily incomplete because developing spinal networks include glutamatergic and cholinergic synaptic connections. During development, the relative balance of the various neurotransmitters systems changes. For example, in the early development of the chick cord, spontaneous activity appears to be most dependent on cholinergic transmission (Milner and Landmesser, 1999). With additional development, GABAergic systems dominate (Chub and O'Donovan, 1998), but, as the chloride equilibrium potential falls, glutamatergic connectivity becomes more important. At the ages we examined in the present work, GABA/glycinergic systems dominate. Indeed, by performing manipulations of extracellular chloride, we were able to demonstrate that the effects on episode duration and interepisode interval could be explained by changes in the inward rate of chloride pumping.
A second shortcoming of the model is that it takes no account of the location of GABA/glycinergic synapses on the neuronal surface. Closely related to this point is the fact that we assumed that the effects of the chloride conductance are exclusively excitatory, but the balance of excitatory and inhibitory effects of GABA depends on the synaptic location (Sernagor et al., 1995; Gulledge and Stuart, 2003). In fact, it has been shown previously that the conductance can sometimes be functionally inhibitory, presumably because of shunting (O'Donovan, 1989; Sernagor et al., 1995; Marchetti et al., 2002).

Finally, we emphasize that this model provides no explanation of the "fast" form of synaptic depression that regulates cycling within the episode. Although we believe that short-term transmitter depletion is the most likely mechanism responsible for this depression, we have no direct evidence to support this idea for neurons participating in rhythmogenesis. It has been shown that primary afferent synapses of the chick cord do exhibit a shortterm synaptic depression (Lee and O'Donovan, 1991) that has been identified with transmitter depletion in the neonatal rat spinal cords (Lev-Tov and Pinco, 1992; Seebach and Mendell, 1996; Li and Burke, 2002). In future experiments, it will be important to investigate the dynamics of synaptic transmission to establish whether this type of depression exists in neurons participating in rhythmogenesis.

\section{Significance of the model}

Our model is consistent with the idea that chloride dynamics play an important role in the genesis of spontaneous activity in the in vitro chick spinal cord between E9 and E11. In addition, it may also explain some of the developmental changes in the nature of spontaneous activity in the developing spinal cord. Early in development, episodes of activity comprise one to two bursts separated by interepisode intervals of 1-2 min. As age increases, both the episode duration and the interepisode interval lengthen (Tabak et al., 2000). A possible explanation from the model could be that, because the surface to volume ratio decreases as cell size increases with development, chloride dynamics slow during both the episode and the interepisode interval. This slowing increases both episode duration and the interepisode interval.

Chloride dynamics may also be important in the spontaneous episodic activity of adult networks. Cohen et al. (2002, 2003) suggested that focal epilepsy of the mesial temporal lobe, characterized by synchronized bursts originating in the subiculum, resulted from a resurgence of developmental features after trauma. In particular, they showed that, in epileptic tissue, some pyramidal cells were depolarized by GABA and that these depolarizing GABA responses contributed to the epileptic activity. Given the similar temporal structure between interepisode epileptic activity of adult network and spontaneous episodic activity in developing networks, it may be that both types of activity are regulated through similar mechanisms, with $\mathrm{Cl}^{-}$homeostasis playing a critical role in both.

\section{References}

Ben Ari Y (2002) Excitatory actions of gaba during development: the nature of the nurture. Nat Rev Neurosci 3:728-739.

Borodinsky LN, Root CM, Cronin JA, Sann SB, Gu X, Spitzer NC (2004) Activity-dependent homeostatic specification of transmitter expression in embryonic neurons. Nature 429:523-530.

Chance FS, Nelson SB, Abbott LF (1998) Synaptic depression and the temporal response characteristics of V1 cells. J Neurosci 18:4785-4799.

Chub N, O’Donovan MJ (1998) Blockade and recovery of spontaneous rhythmic activity after application of neurotransmitter antagonists to spinal networks of the chick embryo. J Neurosci 18:294-306. 
Chub N, O’Donovan MJ (2001) Post-episode depression of GABAergic transmission in spinal neurons of the chick embryo. J Neurophysiol $85: 2166-2176$.

Cohen I, Navarro V, Clemenceau S, Baulac M, Miles R (2002) On the origin of interictal activity in human temporal lobe epilepsy in vitro. Science 298:1418-1421.

Cohen I, Navarro V, Le Duigou C, Miles R (2003) Mesial temporal lobe epilepsy: a pathological replay of developmental mechanisms? Biol Cell 95:329-333.

Delpire E (2000) Cation-chloride cotransporters in neuronal communication. News Physiol Sci 15:309-312.

Fedirchuk B, Wenner P, Whelan PJ, Ho S, Tabak J, O’Donovan MJ (1999) Spontaneous network activity transiently depresses synaptic transmission in the embryonic chick spinal cord. J Neurosci 19:2102-2112.

Feller MB (1999) Spontaneous correlated activity in developing neural circuits. Neuron 22:653-656.

Gu X, Spitzer NC (1995) Distinct aspects of neuronal differentiation encoded by frequency of spontaneous $\mathrm{Ca}^{2+}$ transients. Nature 375:784-787.

Gulledge AT, Stuart GJ (2003) Action potential initiation and propagation in layer 5 pyramidal neurons of the rat prefrontal cortex: absence of dopamine modulation. J Neurosci 23:11363-11372.

Ho S, O’Donovan MJ (1993) Regionalization and inter-segmental coordination of rhythm generating networks in the spinal cord of the chick embryo. J Neurosci 13:1345-1371.

Katz LC, Shatz CJ (1996) Synaptic activity and the construction of cortical circuits. Science 274:1133-1138.

Lee MT, O’Donovan MJ (1991) Organization of hindlimb muscle afferent projections to lumbosacral motoneurons in the chick embryo. J Neurosci 11:2564-2573.

Lev-Tov A, Pinco M (1992) In vitro studies of prolonged synaptic depression in the neonatal rat spinal cord. J Physiol (Lond) 447:149-169.

Li M, Jia M, Fields RD, Nelson PG (1996) Modulation of calcium currents by electrical activity. J Neurophysiol 76:2595-2607.

Li Y, Burke RE (2002) Developmental changes in short-term synaptic depression in the neonatal mouse spinal cord. J Neurophysiol 88:3218-3231.

Majewska MD, Demirgoren S, Spivak CE, London ED (1990) The neurosteroid dehydroepiandrosterone sulfate is an allosteric antagonist of the GABAA receptor. Brain Res 526:143-146.

Marchetti C, Pagnotta S, Donato R, Nistri A (2002) Inhibition of spinal or hypoglossal motoneurons of the newborn rat by glycine or GABA. Eur J Neurosci 15:975-983.

Marchetti C, Tabak J, O’Donovan MJ, Rinzel J (2003) Model of spontaneous activity in the developing spinal cord using activity-dependent variations of intracellular chloride. Soc Nerosci Abstr 29:278.8.

Mienville JM, Vicini S (1989) Pregnenolone sulfate antagonizes GABAA receptor-mediated currents via a reduction of channel opening frequency. Brain Res 489:190-194.

Milner LD, Landmesser LT (1999) Cholinergic and GABAergic inputs drive patterned spontaneous motoneuron activity before target contact. J Neurosci 19:3007-3022.

O'Donovan MJ (1989) Motor activity in the isolated spinal cord of the chick embryo: synaptic drive and firing pattern of single motoneurons. J Neurosci 9:943-958.

Opitz T, De Lima AD, Voigt T (2002) Spontaneous development of synchronous oscillatory activity during maturation of cortical networks in vitro. J Neurophysiol 88:2196-2206.

Pinto DJ, Brumberg JC, Simons DJ, Ermentrout GB (1996) A quantitative population model of whisker barrels: re-examining the Wilson-Cowan equations. J Comput Neurosci 3:247-264.

Rinzel J (1985) Bursting oscillations in an excitable membrane model. In: Ordinary and partial differential equations, Proceedings of the 8th Dundee Conference. Lecture notes in mathematics (Sleeman BD, Jarvis RJ, eds), pp 304-316. Berlin: Springer.

Rinzel J, Ermentrout GB (1998) Analysis of neural excitability and oscillations. In: Methods in neuronal modeling (Koch C, Segev I, eds), pp 251291. Cambridge, MA: MIT.

Russell JM (2000) Sodium-potassium-chloride cotransport. Physiol Rev 80:211-276.

Seebach BS, Mendell LM (1996) Maturation in properties of motoneurons and their segmental input in the neonatal rat. J Neurophysiol 76:3875-3885.

Sernagor E, Chub N, Ritter A, O’Donovan MJ (1995) Pharmacological characterization of the rhythmic synaptic drive onto lumbosacral motoneurons in the chick embryo spinal cord. J Neurosci 15:7452-7464.

Shen W, Mennerick S, Zorumski EC, Covey DF, Zorumski CF (1999) Pregnenolone sulfate and dehydroepiandrosterone sulfate inhibit GABAgated chloride currents in Xenopus oocytes expressing picrotoxininsensitive GABA(A) receptors. Neuropharmacology 38:267-271.

Sherman A, Rinzel J, Keizer J (1988) Emergence of organized bursting in clusters of pancreatic beta-cells by channel sharing. Biophys J 54:411-425.

Strogatz S (1994) Nonlinear dynamics and chaos. Redding, MA: Addison Wesley.

Sung KW, Kirby M, McDonald MP, Lovinger DM, Delpire E (2000) Abnor$\mathrm{mal} \mathrm{GABA}_{\mathrm{A}}$ receptor-mediated currents in dorsal root ganglion neurons isolated from Na-K-2Cl cotransporter null mice. J Neurosci 20:7531-7538.

Tabak J, Senn W, O’Donovan MJ, Rinzel J (2000) Modeling of spontaneous activity in developing spinal cord using activity-dependent depression in an excitatory network. J Neurosci 20:3041-3056.

Tabak J, Rinzel J, O’Donovan MJ (2001) The role of activity-dependent network depression in the expression and self-regulation of spontaneous activity in the developing spinal cord. J Neurosci 21:8966-8978.

Varela JA, Sen K, Gibson J, Fost J, Abbott LF, Nelson SB (1997) A quantitative description of short-term plasticity at excitatory synapses in layer $2 / 3$ of rat primary visual cortex. J Neurosci 17:7926-7940.

Zhang LI, Poo MM (2001) Electrical activity and development of neural circuits. Nat Neurosci [Suppl] 4:1207-1214. 